\title{
INDIRECT STABILIZATION OF LOCALLY COUPLED WAVE-TYPE SYSTEMS
}

\author{
Fatiha Alabau-Boussouira ${ }^{1}$ And Matthieu LÉautaud ${ }^{2,3,4}$
}

\begin{abstract}
We study in an abstract setting the indirect stabilization of systems of two wave-like equations coupled by a localized zero order term. Only one of the two equations is directly damped. The main novelty in this paper is that the coupling operator is not assumed to be coercive in the underlying space. We show that the energy of smooth solutions of these systems decays polynomially at infinity, whereas it is known that exponential stability does not hold (see [F. Alabau, P. Cannarsa and V. Komornik, J. Evol. Equ. 2 (2002) 127-150]). We give applications of our result to locally or boundary damped wave or plate systems. In any space dimension, we prove polynomial stability under geometric conditions on both the coupling and the damping regions. In one space dimension, the result holds for arbitrary non-empty open damping and coupling regions, and in particular when these two regions have an empty intersection. Hence, indirect polynomial stability holds even though the feedback is active in a region in which the coupling vanishes and vice versa.
\end{abstract}

Mathematics Subject Classification. 35L05, 35L51, 93C20, 93D20.

Received March 3, 2010. Revised November 2, 2010.

Published online September 14, 2011.

\section{INTRODUCTION}

\subsection{Motivation and general context}

The decay properties for the energy of a solution of the damped wave equation are well known since the works $[20,26]$ and definitively [11,23]. More precisely, given a bounded open domain $\Omega \subset \mathbb{R}^{N}$ with boundary $\Gamma=\partial \Omega$, we consider either the internally damped wave equation

$$
\begin{cases}u^{\prime \prime}-\Delta u+b u^{\prime}=0 & \text { in }(0,+\infty) \times \Omega, \\ u=0 & \text { on }(0,+\infty) \times \Gamma \\ \left(u, u^{\prime}\right)(0, \cdot)=\left(u^{0}(\cdot), u^{1}(\cdot)\right) & \text { in } \Omega\end{cases}
$$

Keywords and phrases. Stabilization, indirect damping, hyperbolic systems, wave equation.

1 Present position: Délégation CNRS at MAPMO, UMR 6628, Current position: LMAM, Université Paul Verlaine-Metz and CNRS (UMR 7122) Metz Cedex 1, France. alabau@univ-metz.fr

${ }^{2}$ Université Pierre et Marie Curie Paris 6, UMR 7598, Laboratoire Jacques-Louis Lions, 75005 Paris, France.

3 CNRS, UMR 7598 LJLL, 75005 Paris, France. leautaud@ann.jussieu.fr

4 Laboratoire POEMS, INRIA Paris-Rocquencourt/ENSTA, CNRS UMR 2706, 78153 Le Chesnay, France. 
or the boundary damped wave equation

$$
\begin{cases}u^{\prime \prime}-\Delta u=0 & \text { in }(0,+\infty) \times \Omega, \\ \frac{\partial u}{\partial \nu}+l u+b u^{\prime}=0 & \text { on }(0,+\infty) \times \Gamma_{b}, \Gamma_{b} \subset \Gamma, \\ u=0 & \text { on }(0,+\infty) \times\left(\Gamma \backslash \Gamma_{b}\right), \\ \left(u, u^{\prime}\right)(0, \cdot)=\left(u^{0}(\cdot), u^{1}(\cdot)\right) & \text { in } \Omega .\end{cases}
$$

Here, $u=u(t, x), u^{\prime}$ denotes the time derivative of $u$ and $\nu$ stands for the outward unit normal to $\Gamma$. In these two cases, the dissipation is due to the damping term $b u^{\prime}$, where $b=b(x)$ is a non-negative function on $\Omega$ in (1.1), on $\Gamma$ in (1.2). The dissipation of the energy

$$
E(u(t))=\frac{1}{2} \int_{\Omega}\left(\left|u^{\prime}\right|^{2}+|\nabla u|^{2}\right) \mathrm{d} x \quad \text { or } \quad E(u(t))=\frac{1}{2} \int_{\Omega}\left(\left|u^{\prime}\right|^{2}+|\nabla u|^{2}\right) \mathrm{d} x+\frac{1}{2} \int_{\Gamma_{b}} l(x) u^{2} \mathrm{~d} \gamma
$$

is given by

$$
E^{\prime}(u(t))=-\int_{\Omega} b(x)\left|u^{\prime}\right|^{2} \mathrm{~d} x \quad \text { or } \quad E^{\prime}(u(t))=-\int_{\Gamma_{b}} b(x)\left|u^{\prime}\right|^{2} \mathrm{~d} \gamma
$$

respectively in the internal and the boundary damping case. In both cases, the localization of the damping, $\operatorname{supp}(b)$ must satisfy some geometric conditions (see $[11,20,26]$ ) in order for the energy of the solutions to decay exponentially, i.e., so that there exist two constants $M, \kappa>0$ satisfying

$$
E(u(t)) \leq M \mathrm{e}^{-\kappa t} E(u(0)), \quad t>0,
$$

for all initial data $\left(u^{0}, u^{1}\right)$ of finite energy. In the case of internal damping (1.1), the explicit value of the best decay rate $\kappa$ is moreover given in [23].

Besides, when no feedback is applied to the wave equation, i.e., $b=0$ in $\Omega$ in (1.1) or on $\Gamma$ in (1.2), then the energy is conserved, $E(u(t))=E(u(0))$ for every $t>0$.

The question we are interested in is what are the stability properties of the following systems, obtained by coupling an exponentially stable wave equation with a conservative one, that is

$$
\begin{cases}u_{1}^{\prime \prime}-\Delta u_{1}+\delta p u_{2}+b u_{1}^{\prime}=0 & \text { in }(0,+\infty) \times \Omega, \\ u_{2}^{\prime \prime}-\Delta u_{2}+p u_{1}=0 & \text { in }(0,+\infty) \times \Omega, \\ u_{1}=u_{2}=0 & \text { on }(0,+\infty) \times \Gamma, \\ u_{j}(0, \cdot)=u_{j}^{0}(\cdot), u_{j}^{\prime}(0, \cdot)=u_{j}^{1}(\cdot), \quad j=1,2 & \text { in } \Omega,\end{cases}
$$

in the case of internal damping, and

$$
\begin{cases}u_{1}^{\prime \prime}-\Delta u_{1}+\delta p u_{2}=0 & \text { in }(0,+\infty) \times \Omega, \\ u_{2}^{\prime \prime}-\Delta u_{2}+p u_{1}=0 & \text { in }(0,+\infty) \times \Omega, \\ \frac{\partial u_{1}}{\partial \nu}+l u_{1}+b u_{1}^{\prime}=0 & \text { on }(0,+\infty) \times \Gamma_{b}, \Gamma_{b} \subset \Gamma, \\ u_{1}=0 & \text { on }(0,+\infty) \times\left(\Gamma \backslash \Gamma_{b}\right), \\ u_{2}=0 & \text { on }(0,+\infty) \times \Gamma, \\ u_{j}(0, \cdot)=u_{j}^{0}(\cdot), u_{j}^{\prime}(0, \cdot)=u_{j}^{1}(\cdot), j=1,2 & \text { in } \Omega,\end{cases}
$$

in the case of boundary damping. Here, $\delta>0$ is a constant and $p \geq 0$ denotes the coupling coefficient. The case of a constant coupling $p$ has already been treated in [1] for (1.3) (internal damping) and in $[2,3]$ for (1.4) (boundary damping). One key assumption in these three works is that the coupling coefficient satisfies $p(x) \geq C>0$ for all $x \in \Omega$. The main goal of this paper is to generalize these results to cases for which the coupling $p=p(x)$ can vanish in some part of $\Omega$. It seems natural that we shall have to suppose some geometric conditions on the localization of the coupling, that is, on the support of the function $p(x)$. 
The study of systems like (1.3)-(1.4) (and more generally (1.5) below) is motivated by several physical considerations. Indirect damping of reversible systems occurs in many applications in engineering and mechanics. Indeed, it arises whenever it is impossible or too expensive to damp all the components of the state, and it is hence important to study stabilization properties of coupled systems with a reduced number of feedbacks. For finite dimensional systems, this problem is fully understood thanks to the Kalman rank condition. In the case of coupled PDE's, the situation is much more involved. Effectiveness of indirect damping mechanisms depends in a complex way on the assumptions on all the operators involved (i.e. on the operators $A_{1}, A_{2}, P$ and $B$ in system (1.5) below). In [26], Lions considers the case of several different coupled wave-wave or wave-Petrowsky systems and studies partial observability or controllability properties. In [1], the authors give the following example of two elastic membranes subjected to an elastic force that attracts one membrane to the other with coefficient $\kappa>0$,

$$
\begin{cases}u_{1}^{\prime \prime}-\Delta u_{1}+\kappa u_{1}-\kappa u_{2}+\beta u_{1}^{\prime}=0 & \text { in }(0,+\infty) \times \Omega, \\ u_{2}^{\prime \prime}-\Delta u_{2}+\kappa u_{2}-\kappa u_{1}=0 & \text { in }(0,+\infty) \times \Omega, \\ u_{1}=u_{2}=0 & \text { on }(0,+\infty) \times \Gamma, \\ u_{j}(0, \cdot)=u_{j}^{0}(\cdot), u_{j}^{\prime}(0, \cdot)=u_{j}^{1}(\cdot), j=1,2 & \text { in } \Omega .\end{cases}
$$

Another example raised by mechanical applications is the so-called Timoshenko beams. This system consists in two one-dimensional wave equations coupled by first order terms. One can show that polynomial stability holds through indirect mechanisms (see [8,9] and the references therein) such as the ones considered in the present paper. Similar questions arise in the analysis of indirect control of locally coupled parabolic PDE's (see system (1.8) below). We here also point out the works [25] and [14], Section 6, where the exponential and the polynomial stabilization of a coupled hyperbolic-parabolic system of thermoelasticity are addressed with microlocal techniques.

In a more general setting, we are interested in the stability properties of systems of second order evolution equations coupling a conservative equation and an exponentially stable one. The abstract model that we shall refer to is the following

$$
\left\{\begin{array}{l}
u_{1}^{\prime \prime}+A_{1} u_{1}+\delta P u_{2}+B u_{1}^{\prime}=0, \\
u_{2}^{\prime \prime}+A_{2} u_{2}+P^{*} u_{1}=0, \\
\left(u_{j}, u_{j}^{\prime}\right)(0)=\left(u_{j}^{0}, u_{j}^{1}\right), \quad j=1,2,
\end{array}\right.
$$

where $A_{1}$ and $A_{2}$ are positive selfadjoint operators with compact resolvent on an infinite dimensional Hilbert space $H$. The coupling operator $P$ is assumed to be bounded on $H$ and $P^{*}$ is its adjoint. The stabilization operator $B$ will be supposed to be either bounded on $H$ (which corresponds to the case of internal stabilization) or unbounded (which corresponds to the case of boundary stabilization). The total energy of a solution $U=\left(u_{1}, u_{2}\right)$ of (1.5) is defined by

$$
E(U(t))=\frac{1}{2}\left(\left\|u_{1}^{\prime}\right\|_{H}^{2}+\left\|A_{1}^{1 / 2} u_{1}\right\|_{H}^{2}+\delta\left\|u_{2}^{\prime}\right\|_{H}^{2}+\delta\left\|A_{2}^{1 / 2} u_{2}\right\|_{H}^{2}\right)+\delta\left(u_{1}, P u_{2}\right)_{H}
$$

where $(\cdot, \cdot)_{H}$ denotes the inner product on $H$ and $\|\cdot\|_{H}$ the associated norm. Note that we have to consider different operators $A_{j}, j=1,2$, in order to treat the boundary damping case. In the applications to coupled wave equations, $A_{1}$ and $A_{2}$ will be the same Laplace operator, i.e., with the same speed of propagation, but with different boundary conditions. We have moreover to suppose the natural assumption that $\delta$ and $p^{+}=\|P\|_{\mathcal{L}(H)}=\sup \left\{\|P v\|_{H},\|v\|_{H}=1\right\}$ are sufficiently small so that the energy is positive.

The question is now: Is the full system (1.5) stable, and if so, at which rate?

A first answer to this question is that system (1.5) cannot be exponentially stable (see [1]). Indeed, this system is a compact perturbation of the decoupled system (obtained by taking $P=0$ in (1.5)), which is unstable.

Despite this negative result, one can prove polynomial stability properties for certain classes of operators $A_{1}, A_{2}, P, B$. More precisely, under the assumption that the operator $P$ is coercive on $H$, the authors prove in $[1,3,12]$ that the energy decays at least polynomially at infinity. In these papers, the coercivity assumption on $P$ is essential. In the applications we want to address (see $(1.3),(1.4)$ with $p(x)$ locally supported), this assumption clearly fails. For these examples, as well as in the abstract setting (1.5), one has to develop new 
ideas and hypotheses to understand how the damping effect is transmitted from the first equation to the second one. We shall instead suppose that $P$ is only partially coercive (see assumption $(A 1)$ below). For the PDE systems under view (see Sects. 1.2 and 4), this assumption holds under geometric conditions of multiplier type on the support of the coupling coefficient $p$. Similar conditions are required on the support of the damping coefficient $b$, so that we prove polynomial stabilization in several situations in which the damping region and the coupling region have a very small intersection (see Fig. 2 below). In particular in one space dimension, we prove such a result with an empty intersection. This is an important feature since it shows that the information is transferred from the damping region to the coupling region in a sufficient way to stabilize indirectly the second equation, even though these two regions do not meet. In particular, one can notice that in the damping region, the second equation is decoupled from the first one and is conservative in this area (see also Fig. 2 below). More generally, such locally coupled systems of PDE's can be viewed as transmission problems between areas of active/inactive dampings and couplings to areas with inactive/active dampings and couplings.

In this paper, the main result concerning the abstract system (1.5) is a polynomial stability theorem under certain assumptions on the operators $P$ and $B$ (see Thm. 2.4 in the case $B$ bounded and Thm. 2.7 in the case $B$ unbounded). This abstract result can then be applied to a large class of second order evolution equations. In Section 4, we treat the case of two locally coupled wave or plate equations, with an internal or a boundary damping. The problem that first motivated this work is the case (1.3) of partially internally damped wave equations. We now detail the results obtained for this problem, that sum up our study.

\subsection{Results for two coupled wave equations}

In this section, we consider problem (1.3) in a domain $\Omega \subset \mathbb{R}^{N}$ with $\mathcal{C}^{2}$ boundary. The damping function $b$ and the coupling function $p$ are two bounded real valued functions on $\Omega$, satisfying

$$
\left\{\begin{array}{l}
0 \leq b \leq b^{+} \text {and } 0 \leq p \leq p^{+} \text {on } \Omega \\
b \geq b^{-}>0 \text { on } \omega_{b} \\
p \geq p^{-}>0 \text { on } \omega_{p}
\end{array}\right.
$$

for $\omega_{b}$ and $\omega_{p}$ two non-empty open subsets of $\Omega$. As usual for damped wave equations, we have to make some geometric assumptions on the sets $\omega_{b}$ and $\omega_{p}$ so that the energy of a single wave decays sufficiently rapidly at infinity. Here, we shall use the piecewise multipliers geometric condition (PMGC).

Definition 1.1 (PMGC). We say that $\omega \subset \Omega$ satisfies the PMGC if there exist $\Omega_{j} \subset \Omega$ having Lipschitz boundary and $x_{j} \in \mathbb{R}^{N}, j=1 \ldots J$ such that $\Omega_{j} \cap \Omega_{i}=\emptyset$ for $j \neq i$ and $\omega$ contains a neighborhood in $\Omega$ of the set $\bigcup_{j=1}^{J} \gamma_{j}\left(x_{j}\right) \cup\left(\Omega \backslash \bigcup_{j=1}^{J} \Omega_{j}\right)$, where $\gamma_{j}\left(x_{j}\right)=\left\{x \in \partial \Omega_{j},\left(x-x_{j}\right) \cdot \nu_{j}(x)>0\right\}$ and $\nu_{j}$ is the outward unit normal to $\partial \Omega_{j}$.

This geometric assumption was introduced in [27] and further used in [5,6]. It is a generalization of the usual multiplier geometric condition (or $\Gamma$-condition) of $[26,31]$, saying that $\omega$ contains a neighborhood in $\Omega$ of the set $\left\{x \in \partial \Omega,\left(x-x_{0}\right) \cdot \nu(x)>0\right\}$, for some $x_{0} \in \mathbb{R}^{N}$. However, the PMGC is of course much more restrictive than the sharp geometric control condition (GCC) introduced in [11], saying that every generalized geodesic (i.e. ray of geometrical optics) enters the region $\omega$ in finite time. Let us briefly compare these three different geometric conditions on the following simple 2-D example. We consider the case where $\Omega$ is a disk and draw in Figure 1 three different subsets of $\Omega$. The $\Gamma$-condition is only satisfied by $\omega_{0}$, for some $x_{0}$ sufficiently far on the left of the figure. The PMGC is satisfied by $\omega_{0}$, and by $\omega_{1}$ (which contains a neighborhood of a diameter) for some $x_{1}$ on the left and $x_{2}$ on the right, sufficiently far. However, $\omega_{2}$, containing only a neighborhood of a radius, does not satisfy the PMGC, since $\Omega \backslash \bar{\omega}_{2}$ is connected, so that the PMGC is reduced in this case to the $\Gamma$-condition (which is here not satisfied). Finally, the GCC is satisfied by these three different subsets of $\Omega$.

In other geometric situations, the $\Gamma$-condition and the GCC are further compared in [11], pp. 1030-1032. In particular (in the context of boundary stabilization), the authors provide several 2-D examples in which the GCC is satisfied but not the $\Gamma$-condition. In [29], Remark 6, the author proves directly from a geometrical point 

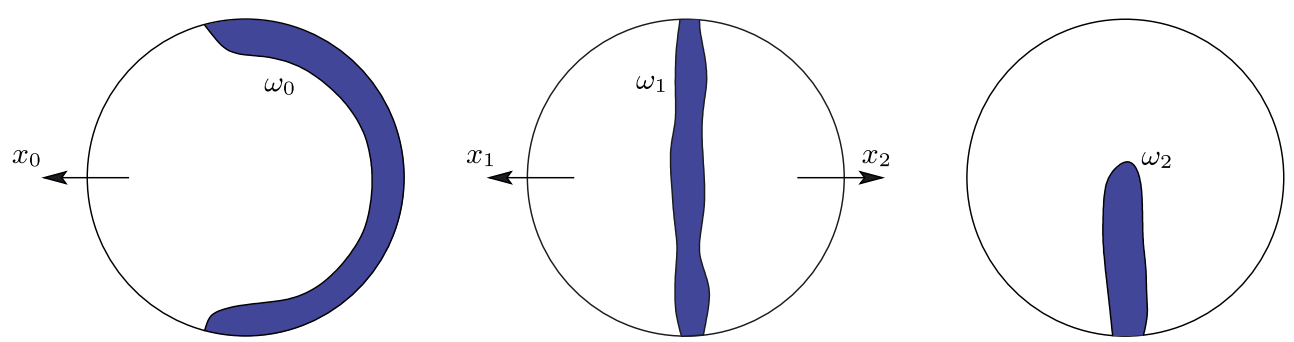

Figure 1. Comparison between the different geometric conditions in the disk.

of view that the GCC implies the PMGC (and more generally, that the GCC implies any multiplier condition). Then, in [29], Section 5, he describes in dimension two different situations for which GCC holds but neither the $\Gamma$-condition nor the PMGC do. To conclude this comparison, let us note that in one space dimension, the PMGC is equivalent to the GCC, and is satisfied by every nonempty subset of the interval $\Omega$.

We denote by $\lambda$ the smallest eigenvalue of the Laplace operator on $\Omega$, with Dirichlet boundary conditions. We also have the identity $\lambda=1 / C_{\mathcal{P}}^{2}$, where $C_{\mathcal{P}}$ is the Poincaré's constant of $\Omega$. Note that according to (1.6), the energy of a solution $U$ of (1.3) is defined by

$$
E(U(t))=\frac{1}{2}\left(\left\|u_{1}^{\prime}\right\|_{L^{2}(\Omega)}^{2}+\left\|\nabla u_{1}\right\|_{L^{2}(\Omega)}^{2}+\delta\left\|u_{2}^{\prime}\right\|_{L^{2}(\Omega)}^{2}+\delta\left\|\nabla u_{2}\right\|_{L^{2}(\Omega)}^{2}\right)+\delta\left(p u_{1}, u_{2}\right)_{L^{2}(\Omega)},
$$

and is positive as soon as $p^{+}<\lambda$ and $0<\delta<\frac{\lambda}{p^{+}}$. Finally, for $n \in \mathbb{N}$, we denote by $\mathcal{D}\left(\left(-\Delta_{D}\right)^{\frac{n}{2}}\right)$ the domain of the Laplace operator with Dirichlet boundary conditions to the power $\frac{n}{2}$. We recall that we have for instance

$$
\begin{array}{ll}
\mathcal{D}\left(\left(-\Delta_{D}\right)^{\frac{n}{2}}\right)=\left\{v \in H^{n}(\Omega), v=0 \text { on } \partial \Omega\right\} & \text { for } n=1,2, \\
\mathcal{D}\left(\left(-\Delta_{D}\right)^{\frac{n}{2}}\right)=\left\{v \in H^{n}(\Omega), v=0 \text { and } \Delta v=0 \text { on } \partial \Omega\right\} & \text { for } n=3,4, \text { etc. }
\end{array}
$$

With this notation, we can state the stability theorem for system (1.3).

\section{Theorem 1.2.}

(i) Suppose that $\omega_{b}$ and $\omega_{p}$ satisfy the PMGC. Then there exists $p_{*} \in(0, \lambda]$ such that for all $0<p^{+}<p_{*}$ there exists $\delta_{*}=\delta_{*}\left(p^{+}, p^{-}\right) \in\left(0, \frac{\lambda}{p^{+}}\right]$, such that for all $\delta \in\left(0, \delta_{*}\right)$ and all $b, p \in W^{q, \infty}(\Omega)$ satisfying $(1.7)$, the solution $U=\left(u_{1}, u_{2}, u_{1}^{\prime}, u_{2}^{\prime}\right)$ of (1.3) satisfies for $n \in \mathbb{N}, n \leq q$, for some $C_{n}>0$,

$E(U(t)) \leq \frac{C_{n}}{t^{n}} \sum_{i=0}^{n} E\left(U^{(i)}(0)\right) \quad \forall t>0, U^{0}=\left(u_{1}^{0}, u_{2}^{0}, u_{1}^{1}, u_{2}^{1}\right) \in \mathcal{D}\left(\left(-\Delta_{D}\right)^{\frac{n+1}{2}}\right)^{2} \times \mathcal{D}\left(\left(-\Delta_{D}\right)^{\frac{n}{2}}\right)^{2}$

Besides, if $U^{0} \in\left(H_{0}^{1}\right)^{2} \times\left(L^{2}\right)^{2}$, then $E(U(t))$ converges to zero as $t$ goes to infinity.

(ii) If moreover either $\omega_{b} \subset \omega_{p}$ or $\omega_{p} \subset \omega_{b}$, then the result holds for $\delta_{*}=\frac{\lambda}{p^{+}}$.

This theorem is a consequence of Theorem 2.4 below. The fact that problem (1.3) satisfies the assumptions of Theorem 2.4 is postponed in Section 4.1. Note that the constants $p_{*}$ and $\delta_{*}$ are explicit functions of the parameters of the problem and of the constants coming from the multiplier method. Moreover, the constants $C_{n}$ depend on $\delta, p^{+}, p^{-}, b^{+}$and $b^{-}$. The smoothness assumption on the coefficients $p$ and $b$ comes from Lemma 2.6. If these parameters are not smooth, Theorem 1.2 is still valid for initial data in $\mathcal{D}\left(\mathcal{A}_{P, \delta}^{n}\right)$ where the operator $\mathcal{A}_{P, \delta}$ is defined in (2.4) below. However, in this case, we cannot explicit the space $\mathcal{D}\left(\mathcal{A}_{P, \delta}^{n}\right)$ in terms of classical Sobolev spaces.

Let us draw in Figure 2 some geometric situations covered by Theorem $1.2(i)$. 


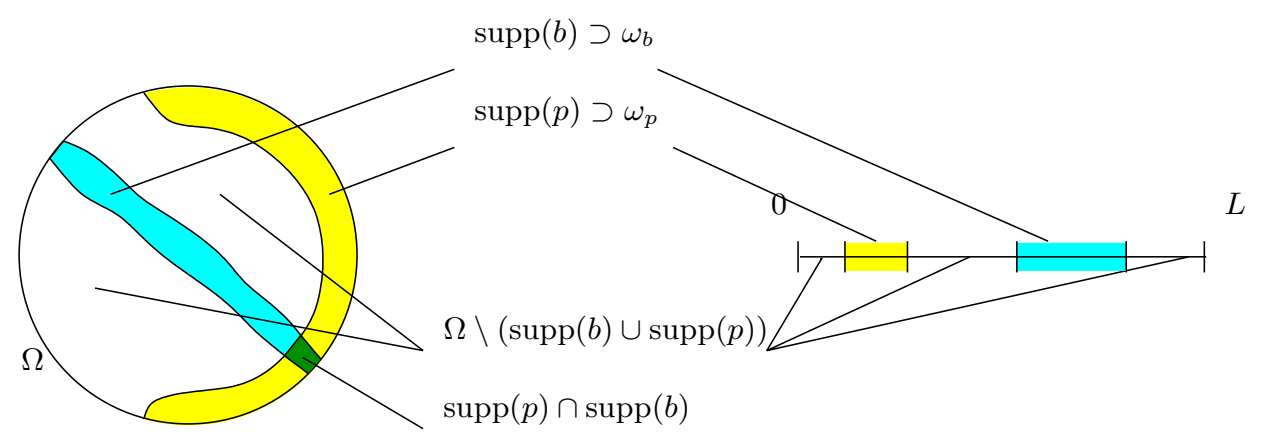

Figure 2. Some one and two dimensional geometric situations covered by Theorem $1.2(i)$.

Locally, the equations satisfied by $u_{1}$ and $u_{2}$ are

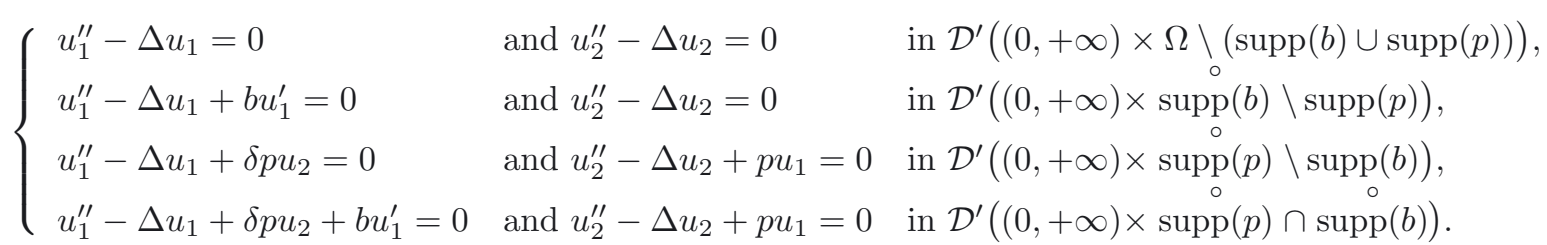

In particular, in $\Omega \backslash(\operatorname{supp}(b) \cup \operatorname{supp}(p))$, the states $u_{1}$ and $u_{2}$ are decoupled and both satisfy a conservative equation. Besides, the subset of $\Omega$ where $u_{1}$ and $u_{2}$ are coupled and $u_{1}$ is damped, i.e. $\operatorname{supp}(p) \cap \operatorname{supp}(b)$, might be reduced to the empty set, as it is the case in the one dimensional example.

Some comments can be made about Theorem 1.2. One particularly interesting question for this type of coupled problem is the case $\omega_{b} \cap \omega_{p}=\emptyset$. This question first arised in the field of control theory for coupled evolution equations, and, to the authors' knowledge, is still unsolved. More precisely, consider the parabolic system

$$
\begin{cases}u_{1}^{\prime}-\Delta u_{1}+\delta p u_{2}=\mathbb{1}_{\omega_{b}} f & \text { in }(0, T) \times \Omega, \\ u_{2}^{\prime}-\Delta u_{2}+p u_{1}=0 & \text { in }(0, T) \times \Omega, \\ u_{1}=u_{2}=0 & \text { on }(0, T) \times \partial \Omega \\ u_{1}(0, \cdot)=u_{1}^{0}, u_{2}(0, \cdot)=u_{2}^{0} & \text { in } \Omega,\end{cases}
$$

or its hyperbolic counterpart

$$
\begin{cases}u_{1}^{\prime \prime}-\Delta u_{1}+\delta p u_{2}=\mathbb{1}_{\omega_{b}} f & \text { in }(0, T) \times \Omega \\ u_{2}^{\prime \prime}-\Delta u_{2}+p u_{1}=0 & \text { in }(0, T) \times \Omega \\ u_{1}=u_{2}=0 & \text { on }(0, T) \times \partial \Omega \\ u_{1}(0, \cdot)=u_{1}^{0}, u_{2}(0, \cdot)=u_{2}^{0} & \text { in } \Omega \\ u_{1}^{\prime}(0, \cdot)=u_{1}^{1}, u_{2}^{\prime}(0, \cdot)=u_{2}^{1} & \text { in } \Omega\end{cases}
$$

where the function $p$ and the subset $\omega_{b}$ are the same as in the stabilization problem. In these two cases, the null-controllability problem under interest is the following: given a positive time $T$ and initial data, is it possible to find a control function $f$ so that the state has been driven to zero in time $T$ ? The parabolic null-controllability problem (1.8) is fully solved in the case $\omega_{b} \cap \omega_{p} \neq \emptyset$ (see $[10,16,17,22]$ ). However, this problem is still open in the case $\omega_{b} \cap \omega_{p}=\emptyset$. Only the approximate controllability has been proved in [19] in this case for $\delta=0$. Concerning the hyperbolic null-controllability problem (1.9), only the case of constant coupling $p$ have been considered, to our knowledge [30] (and [4] for a boundary control).

The second reason for which the case $\omega_{b} \cap \omega_{p}=\emptyset$ is of particular interest in the stabilization problem (1.3) is that, in this case, we don't even know if the strong stability property holds, i.e., if the energy goes to zero 
as $t$ goes to infinity. To our knowledge, the only strong (and logarithmic) stability result for system (1.3) is the following one (we suppose here that $p$ and $b$ are smooth).

Proposition 1.3. Suppose that $\omega_{b} \cap \omega_{p} \neq \emptyset$. Then, the solution $U=\left(u_{1}, u_{2}, u_{1}^{\prime}, u_{2}^{\prime}\right)$ of (1.3) satisfies for $n \in \mathbb{N}$, for some $C_{n}>0$,

$$
E(U(t)) \leq \frac{C_{n}}{\log (2+t)^{n}} \sum_{i=0}^{n} E\left(U^{(i)}(0)\right) \quad \forall t>0
$$

for all initial data $U^{0}=\left(u_{1}^{0}, u_{2}^{0}, u_{1}^{1}, u_{2}^{1}\right) \in \mathcal{D}\left(\left(-\Delta_{D}\right)^{\frac{n+1}{2}}\right)^{2} \times \mathcal{D}\left(\left(-\Delta_{D}\right)^{\frac{n}{2}}\right)^{2}$.

This is a direct consequence of the interpolation inequality for the associated elliptic system, proved in [22], Proposition 5.1, together with the resolvent estimates of [23,24] (see also [13] to obtain the exact power $n$ ). Note that it is natural that logarithmic stability holds without geometric control conditions on the sets $\omega_{b}$ and $\omega_{p}$. However the interpolation inequality for the associated elliptic problem is not known in the case $\omega_{b} \cap \omega_{p}=\emptyset$ and even the strong stability is open.

Now, concerning the stability Theorem 1.2, it first has to be noted that, in dimension $N \geq 2$ the assumption that both $\omega_{b}$ and $\omega_{p}$ satisfy the PMGC implies that $\omega_{b} \cap \omega_{p} \neq \emptyset$ (whereas this is not the case if $\omega_{b}$ and $\omega_{p}$ satisfy the optimal condition of [11]). This theorem is hence of particular interest in dimension $N=1$ (see Fig. 2). In this case, $\Omega=(0, L)$ for some $L>0$, and any non-empty open subinterval $\omega$ satisfies the PMGC. As a consequence, we obtain the following corollary of Theorem 1.2 point $(i)$.

Corollary 1.4. Suppose that $\Omega=(0, L)$. Then, for any non-empty subsets $\omega_{b} \subset \Omega$ and $\omega_{p} \subset \Omega$ (i.e., for any non-vanishing non-negative functions $p$ and $b)$, there exists $p_{*} \in(0, \lambda]$ such that for all $0<p^{+}<p_{*}$ there exists $\delta_{*}=\delta_{*}\left(p^{+}, p^{-}\right) \in\left(0, \frac{\lambda}{p^{+}}\right]$, such that for all $\delta \in\left(0, \delta_{*}\right)$ and all $b, p$ sufficiently smooth, satisfying (1.7), the polynomial stability result of Theorem 1.2 holds.

In particular, this yields in this case a strong (and logarithmic) stability result with $\omega_{b} \cap \omega_{p}=\emptyset$, improving Proposition 1.3. Moreover, this can be a first step to address first the hyperbolic control problem (1.9) and then its parabolic counterpart (1.8) in the case $\omega_{b} \cap \omega_{p}=\emptyset$. Similarly, Theorem 4.4 on boundary stabilization can be a first step to solve the hyperbolic coupled problem controlled from the boundary (to our knowledge only adressed in [4] in the case of constant coupling $p$ ). Then, it could allow one to solve its parabolic counterpart, also controlled from the boundary, which seems to be widely open in several space dimensions.

To conclude this section, let us come back to the geometric assumptions we make. The PMGC does not seem to be necessary or optimal in these problems, but only technical. All the results we state in these paper should hold true (and would be also very interesting in several space dimensions) under the GCC. Replacing PGMC by GCC in all our results would be possible provided that we are able to prove assumptions $(A 2)$ and $(A 3)$ below with microlocal techniques, what we did not yet manage to do.

Remark 1.5. In the sequel, $C$ will denote a generic constant, whose value may change from line to line. Writing $C=C(p, \beta, \ldots)$ or $C=C_{p, \beta, \ldots}$ means that this constant depends on the parameters $p, \beta, \ldots$

\section{Abstract formulation AND MAin RESUlts}

\subsection{Abstract setting and well-posedness}

Let $H$ and $V_{j}, j=1,2$, be infinite dimensional Hilbert spaces such that the injections $V_{j} \subset H$ are dense and compact. We identify $H$ with its dual space and denote by $V_{j}^{\prime}$ the dual space of $V_{j}$, so that the injections $V_{j} \subset H \subset V_{j}^{\prime}$ are dense and compact. We denote by $(\cdot, \cdot)_{H}$ (resp. $(\cdot, \cdot)_{V_{j}}$ ) the inner product on $H$ (resp. $V_{j}$ ), $\|\cdot\|_{H}$ (resp. $\|\cdot\|_{V_{j}}$ ) the associated norm and $\|\cdot\|_{V_{j}^{\prime}}$ the norm on $V_{j}^{\prime}$. Moreover, we write $\langle\cdot, \cdot\rangle_{V_{j}^{\prime}, V_{j}}$ the duality product and $A_{j}$ the duality mapping from $V_{j}$ to $V_{j}^{\prime}$ defined by

$$
\left\langle A_{j} v, w\right\rangle_{V_{j}^{\prime}, V_{j}}=(v, w)_{V_{j}} \quad \forall v, w \in V_{j} .
$$


By abuse of notation, we also write $A_{j}$ the unbounded operator on $H$ with domain

$$
\mathcal{D}\left(A_{j}\right)=\left\{v \in V_{j}, A_{j} v \in H\right\} \subset V_{j} \subset H,
$$

defined by

$$
A_{j}: v \in \mathcal{D}\left(A_{j}\right) \subset H \longrightarrow A_{j} v \in H
$$

In this setting, each operator $A_{j}$ is positive selfadjoint in $H$ and has a compact resolvent.

We shall moreover assume that the subspace $V_{2}$ is continuously imbedded in $V_{1}$, so that we have the following scheme:

$$
V_{2} \hookrightarrow V_{1} \hookrightarrow H \hookrightarrow V_{1}^{\prime} \hookrightarrow V_{2}^{\prime}
$$

where the first and the last inclusions are continuous and the two central ones are dense and compact. We denote $i: V_{2} \hookrightarrow V_{1}$ the natural injection and $\Pi_{V}: V_{1} \mapsto V_{2}$ the natural projection from $V_{1}$ to $V_{2}$. We recall that for $u_{1} \in V_{1}, \Pi_{V} u_{1}$ is characterized by

$$
\left\{\begin{array}{c}
\left\langle A_{1} i\left(\Pi_{V} u_{1}\right), i(\phi)\right\rangle_{V_{1}^{\prime}, V_{1}}=\left\langle A_{1} u_{1}, i(\phi)\right\rangle_{V_{1}^{\prime}, V_{1}} \quad \forall \phi \in V_{2}, u_{1} \in V_{1}, \\
\text { and } \Pi_{V} u_{1} \in V_{2} .
\end{array}\right.
$$

Moreover the operators $i, A_{1}$ and $A_{2}$ are linked by

$$
\left\langle A_{2} \phi, \psi\right\rangle_{V_{2}^{\prime}, V_{2}}=(\phi, \psi)_{V_{2}}=(i(\phi), i(\psi))_{V_{1}}=\left\langle A_{1} i(\phi), i(\psi)\right\rangle_{V_{1}^{\prime}, V_{1}} \quad \forall \phi, \psi \in V_{2},
$$

thanks to the definitions of $A_{1}, A_{2}, i$ and to assumption (2.1). Note also that under these assumptions, the unbounded operators $A_{1}$ and $A_{2}$ coincide on $\mathcal{D}\left(A_{1}\right) \cap \mathcal{D}\left(A_{2}\right)$.

The coupling operator $P$ is a bounded operator on $H$ and $P^{*}$ is its adjoint, $\|P\|_{\mathcal{L}(H)}=p^{+}$. The damping operator $B$ will be supposed to be at least bounded from $V_{1}$ to $V_{1}^{\prime}$ and symmetric non-negative:

$$
\langle B v, w\rangle_{V_{1}^{\prime}, V_{1}}=\langle B w, v\rangle_{V_{1}^{\prime}, V_{1}}, \quad\langle B v, v\rangle_{V_{1}^{\prime}, V_{1}} \geq 0 \quad \forall v, w \in V_{1} .
$$

We denote by $\lambda_{j}, j=1,2$, the largest constant satisfying

$$
\|v\|_{V_{j}}^{2} \geq \lambda_{j}\|v\|_{H}^{2} \quad \forall v \in V_{j}
$$

that is, the smallest eigenvalue of the selfadjoint positive operator $A_{j}$.

Let us study now the abstract system (1.5). This linear evolution equation can be rewritten under the form

$$
\left\{\begin{array}{l}
U^{\prime}+\mathcal{A}_{P, \delta} U=0 \\
U(0)=U^{0} \in \mathcal{H}
\end{array}\right.
$$

where $\mathcal{H}=V_{1} \times V_{2} \times H^{2}$

$$
U=\left(\begin{array}{l}
u_{1} \\
u_{2} \\
v_{1} \\
v_{2}
\end{array}\right), U^{0}=\left(\begin{array}{l}
u_{1}^{0} \\
u_{2}^{0} \\
u_{1}^{1} \\
u_{2}^{1}
\end{array}\right), \mathcal{A}_{P, \delta}=\left(\begin{array}{cccc}
0 & 0 & -\mathrm{Id} & 0 \\
0 & 0 & 0 & -\mathrm{Id} \\
A_{1} & \delta P & B & 0 \\
P^{*} & A_{2} & 0 & 0
\end{array}\right), \mathcal{D}\left(\mathcal{A}_{P, \delta}\right)=\left\{U \in \mathcal{H}, \mathcal{A}_{P, \delta} U \in \mathcal{H}\right\}
$$

We recall that the energy of this system is given by

$$
\begin{aligned}
E(U(t)) & =\frac{1}{2}\left(\left\|v_{1}\right\|_{H}^{2}+\left\|u_{1}\right\|_{V_{1}}^{2}+\delta\left\|v_{2}\right\|_{H}^{2}+\delta\left\|u_{2}\right\|_{V_{2}}^{2}\right)+\delta\left(u_{1}, P u_{2}\right)_{H} \\
& =\frac{1}{2}\left(\left\|u_{1}^{\prime}\right\|_{H}^{2}+\left\|u_{1}\right\|_{V_{1}}^{2}+\delta\left\|u_{2}^{\prime}\right\|_{H}^{2}+\delta\left\|u_{2}\right\|_{V_{2}}^{2}\right)+\delta\left(u_{1}, P u_{2}\right)_{H}
\end{aligned}
$$


and we will require this energy to be positive for any solution $U(t)$. We have the lower bound for the energy

$$
\begin{aligned}
E(U) & \geq \frac{1}{2}\left(\left\|u_{1}^{\prime}\right\|_{H}^{2}+\lambda_{1}\left\|u_{1}\right\|_{H}^{2}+\delta\left\|u_{2}^{\prime}\right\|_{H}^{2}+\delta \lambda_{2}\left\|u_{2}\right\|_{H}^{2}\right)-\frac{\delta p^{+}}{2}\left(\left\|u_{1}\right\|_{H}^{2}+\left\|u_{2}\right\|_{H}^{2}\right) \\
& \geq \frac{1}{2}\left(\left\|u_{1}^{\prime}\right\|_{H}^{2}+\delta\left\|u_{2}^{\prime}\right\|_{H}^{2}\right)+\frac{1}{2}\left(\lambda_{1}-\delta p^{+}\right)\left\|u_{1}\right\|_{H}^{2}+\frac{\delta}{2}\left(\lambda_{2}-p^{+}\right)\left\|u_{2}\right\|_{H}^{2} .
\end{aligned}
$$

Therefore, in the sequel, we shall suppose

$$
0<p^{+}<\lambda_{2} \text {, and } 0<\delta<\frac{\lambda_{1}}{p^{+}}
$$

so that (2.6) holds with positive constants, i.e., $E$ is a positive energy that measures the whole state $U$.

Remark 2.1. Note that for any $\delta>0$, the operator $\left(\begin{array}{cc}A_{1} & \delta P \\ P^{*} & A_{2}\end{array}\right)$ is selfadjoint on the space $H \times H$ endowed with the weighted inner product $(u, v)_{\delta}=\left(u_{1}, v_{1}\right)_{H}+\delta\left(u_{2}, v_{2}\right)_{H}$ (which is the energy space). This operator is moreover positive under the condition (2.7). In the case $B=0$, the operator $\mathcal{A}_{P, \delta}$ is skewadjoint and thus generates a group.

Under the assumptions made above, the system (2.3) (and thus, (1.5)) is well-posed in the sense of semigroup theory.

Proposition 2.2. For all $0 \leq p^{+}<\lambda_{2}$ and $0 \leq \delta<\frac{\lambda_{1}}{p^{+}}$, the operator $\mathcal{A}_{P, \delta}$ is maximal monotone on $\mathcal{H}$. As a consequence, for every $U^{0} \in \mathcal{H}$, problem $(2.3)$ has a unique solution $U \in \mathcal{C}^{0}([0,+\infty) ; \mathcal{H})$. If in addition, $U^{0} \in \mathcal{D}\left(\mathcal{A}_{P, \delta}^{k}\right)$, for some $k \in \mathbb{N}$, then, the solution $U$ is in $\bigcap_{j=0}^{k} \mathcal{C}^{k-j}\left([0,+\infty) ; \mathcal{D}\left(\mathcal{A}_{P, \delta}^{j}\right)\right)$. Moreover, the energy $E(U)$ of the solution defined by $(2.5)$ is locally absolutely continuous, and for strong solutions, i.e., when $U^{0} \in$ $\mathcal{D}\left(\mathcal{A}_{P, \delta}\right)$, we have

$$
E^{\prime}(U(t))=-\left\langle B u_{1}^{\prime}, u_{1}^{\prime}\right\rangle_{V_{1}^{\prime}, V_{1}}
$$

\subsection{Main results}

In all the following, we have to suppose some additional assumptions on the operators $P$ and $B$, in order to prove the stability results. Let us first precise assumptions $(A 1)$ and $(A 2)$, related with the operator $P$. We assume that $P$ is partially coercive, i.e.,

$$
(A 1)\left\{\begin{array}{c}
\text { there exists an operator } \Pi_{P} \in \mathcal{L}(H),\left\|\Pi_{P}\right\|_{\mathcal{L}(H)}=1, \text { and a number } p^{-}>0 \\
\text { such that } \quad(P v, v)_{H} \geq p^{-}\left\|\Pi_{P} v\right\|_{H}^{2} \quad \forall v \in H .
\end{array}\right.
$$

Note that $p^{-} \leq p^{+}=\|P\|_{\mathcal{L}(H)}$ and that $(A 1)$ implies that the operators $P$ and $P^{*}$ are non-negative. We shall moreover make the following assumption $(A 2)$ on one decoupled equation, without damping, but with a right hand-side:

$$
(A 2)\left\{\begin{array}{l}
\exists \alpha_{2}, \beta_{2}, \gamma_{2}>0 \text { such that for all } f_{2} \in \mathcal{C}^{1}([0,+\infty) ; H) \text { and all } 0 \leq S \leq T, \\
\text { the solution } u_{2} \text { of } \\
\quad u_{2}^{\prime \prime}+A_{2} u_{2}=f_{2} \text { in } V_{2}^{\prime}, \\
\quad\left(u_{2}, u_{2}^{\prime}\right)(0)=\left(u_{2}^{0}, u_{2}^{1}\right) \in V_{2} \times H, \\
\text { satisfies, with } e_{2}(t)=1 / 2\left(\left\|u_{2}^{\prime}\right\|_{H}^{2}+\left\|u_{2}\right\|_{V_{2}}^{2}\right), \text { the inequality } \\
\int_{S}^{T} e_{2}(t) \mathrm{d} t \leq \alpha_{2}\left(e_{2}(S)+e_{2}(T)\right)+\beta_{2} \int_{S}^{T}\left\|f_{2}(t)\right\|_{H}^{2} \mathrm{~d} t+\gamma_{2} \int_{S}^{T}\left\|\Pi_{P} u_{2}^{\prime}(t)\right\|_{H}^{2} \mathrm{~d} t .
\end{array}\right.
$$


This corresponds to the second equation in which the coupling term is viewed as a forcing term. This type of estimate will be proved in the applications below by means of multiplier estimates (for a single equation with a right hand-side). Note that the operator $\Pi_{P}$ involved in the estimate of assumption $(A 2)$ is the operator given by assumption $(A 1)$.

Concerning the operator $B$, we shall make the following "stability" assumption for a single damped equation:

$$
(A 3)\left\{\begin{array}{l}
\exists \alpha_{1}, \beta_{1}, \gamma_{1}>0 \text { such that for all } f_{1} \in \mathcal{C}^{1}([0,+\infty) ; H) \text { and all } 0 \leq S \leq T, \\
\text { the solution } u_{1} \text { of } \\
\quad u_{1}^{\prime \prime}+A_{1} u_{1}+B u_{1}^{\prime}=f_{1} \text { in } V_{1}^{\prime}, \\
\quad\left(u_{1}, u_{1}^{\prime}\right)(0)=\left(u_{1}^{0}, u_{1}^{1}\right) \in V_{1} \times H, \\
\text { satisfies, with } e_{1}(t)=1 / 2\left(\left\|u_{1}^{\prime}\right\|_{H}^{2}+\left\|u_{1}\right\|_{V_{1}}^{2}\right), \text { the inequality } \\
\int_{S}^{T} e_{1}(t) \mathrm{d} t \leq \alpha_{1}\left(e_{1}(S)+e_{1}(T)\right)+\beta_{1} \int_{S}^{T}\left\|f_{1}(t)\right\|_{H}^{2} \mathrm{~d} t+\gamma_{1} \int_{S}^{T}\left\langle B u_{1}^{\prime}, u_{1}^{\prime}\right\rangle_{V^{\prime}, V} \mathrm{~d} t .
\end{array}\right.
$$

Remark 2.3. Assumption $(A 3)$ implies in particular that the single damped equation is exponentially stable, since for $f_{1}=0$, we deduce that $e_{1}(t)$, which is locally absolutely continuous and nonincreasing, satisfies the classical integral inequality (see $[18,20]$ ),

$$
\int_{S}^{T} e_{1}(t) \mathrm{d} t \leq\left(2 \alpha_{1}+\gamma_{1}\right) e_{1}(S) \quad \forall 0 \leq S \leq T .
$$

The next assumption we make on the damping operator $B$ depends on its boundedness.

\subsubsection{The case B bounded}

In the bounded case, we shall moreover suppose

$$
\|B\|_{\mathcal{L}(H)}=b^{+} \quad \text { and } \quad V_{2}=V_{1}=V .
$$

As a consequence, we have

$$
A_{1}=A_{2}=A, \quad \lambda_{1}=\lambda_{2}=\lambda, \quad \text { and } \quad i=\Pi_{V}=\operatorname{Id}_{V_{1}} .
$$

The positivity condition (2.7) for the energy becomes

$$
0<p^{+}<\lambda, \quad \text { and } \quad 0<\delta<\frac{\lambda}{p^{+}}
$$

The main result here is:

\section{Theorem 2.4.}

(i) Suppose $(A 1),(A 2),(A 3),(A 4 b)$. Then there exists $p_{*} \in(0, \lambda]$ such that for all $0<p^{+}<p_{*}$ there exists $\delta_{*}=\delta_{*}\left(p^{+}, p^{-}\right) \in\left(0, \frac{\lambda}{p^{+}}\right]$, such that for all $\delta \in\left(0, \delta_{*}\right)$, the solution $U(t)=\exp \left(-\mathcal{A}_{P, \delta} t\right) U^{0}$ of $(1.5)$ satisfies for some $C_{n}>0$,

$$
E(U(t)) \leq \frac{C_{n}}{t^{n}} \sum_{i=0}^{n} E\left(U^{(i)}(0)\right) \quad \forall t>0, U^{0} \in \mathcal{D}\left(\mathcal{A}_{P, \delta}^{n}\right)
$$

Besides, if $U^{0} \in \mathcal{H}$, then $E(U(t))$ converges to zero as $t$ goes to infinity. 
(ii) Suppose moreover either

$$
\|P v\|_{H}^{2} \leq p^{+}(P v, v)_{H} \quad \text { and }(B v, v)_{H} \leq \frac{b^{+}}{p^{-}}(P v, v)_{H}, \quad \forall v \in H,
$$

or

$$
\text { there exists } b^{-}>0 \text { such that }(P v, v)_{H} \leq \frac{p^{+}}{b^{-}}(B v, v)_{H}, \quad \forall v \in H .
$$

Then the result holds for $\delta_{*}=\frac{\lambda}{p^{+}}$.

Remark 2.5. In case ( $i i)$ of Theorem 2.4, the conclusion is much stronger than in case $(i)$. As one sees in the proof below, $\delta_{*}$ is very small in case $(i)$, whereas in case $(i i)$, the result holds for a large panel of $\delta$, including the interval $(0,1]$. More precisely, the constants $p_{*}$ and $\delta_{*}$ are explicit, that is,

- $p_{*}=\min \left\{\frac{1}{2 \gamma_{2}}, \lambda\right\}$ and $\delta_{*}=\delta_{*}\left(p^{+}, p^{-}\right)=\min \left\{\frac{\lambda}{2 \beta_{2}\left(p^{+}\right)^{2} p^{-}}, \frac{\lambda p^{-}}{2 \beta_{1}\left(p^{+}\right)^{2}}, \frac{\lambda}{p^{+}}\right\}$in $(i)$;

- $p_{*}=\min \left\{\frac{1}{2 \beta_{1}}, \lambda\right\}$ and $\delta_{*}=\frac{\lambda}{p^{+}}$in the first case of $(i i)$;

- $p_{*}=\min \left\{\frac{1}{2 \beta_{1}}, \frac{1}{2 \beta_{2}}, \lambda\right\}$ and $\delta_{*}=\frac{\lambda}{p^{+}}$in the second case of $(i i)$.

Note that the question whether our stability result can be extended to $\delta=1$ (i.e. for the symmetric coupled system) in case $(i)$ remains open.

In this case, we are moreover able to give a simple characterization of the space $\mathcal{D}\left(\mathcal{A}_{P, \delta}^{n}\right)$, in terms of the spaces $\mathcal{D}\left(A^{n}\right)$, which is useful in the applications. More precisely, setting $\mathcal{H}_{n}=\left(\mathcal{D}\left(A^{\frac{n+1}{2}}\right)\right)^{2} \times\left(\mathcal{D}\left(A^{\frac{n}{2}}\right)\right)^{2} \subset \mathcal{H}$, we prove the following lemma, inspired by [1], Lemma 3.1.

Lemma 2.6. Suppose that for every $0<k \leq n-1$ (no assumption if $n=1$ ), we have

$$
P \mathcal{D}\left(A^{\frac{k+1}{2}}\right) \subset \mathcal{D}\left(A^{\frac{k}{2}}\right), \quad P^{*} \mathcal{D}\left(A^{\frac{k+1}{2}}\right) \subset \mathcal{D}\left(A^{\frac{k}{2}}\right) \quad \text { and } \quad B \mathcal{D}\left(A^{\frac{k+1}{2}}\right) \subset \mathcal{D}\left(A^{\frac{k}{2}}\right)
$$

Then $\mathcal{H}_{k}=\mathcal{D}\left(\mathcal{A}_{P, \delta}^{k}\right)$ for every $0 \leq k \leq n$.

Proof. We proceed by induction on $n \in \mathbb{N}$. In the case $n=1$, there is no need of an additional assumption since the operators $B$ and $P$ are bounded on $H$. Now, we assume that $\mathcal{H}_{n-1}=\mathcal{D}\left(\mathcal{A}_{P, \delta}^{n-1}\right)$ and prove $\mathcal{H}_{n}=\mathcal{D}\left(\mathcal{A}_{P, \delta}^{n}\right)$. We have

$$
\begin{aligned}
\mathcal{D}\left(\mathcal{A}_{P, \delta}^{n}\right)= & \left\{U \in \mathcal{D}\left(\mathcal{A}_{P, \delta}^{n-1}\right), \mathcal{A}_{P, \delta} U \in \mathcal{D}\left(\mathcal{A}_{P, \delta}^{n}\right)\right\} \\
= & \left\{\left(u_{1}, u_{2}, v_{1}, v_{2}\right) \in\left(\mathcal{D}\left(A^{\frac{n}{2}}\right)\right)^{2} \times\left(\mathcal{D}\left(A^{\frac{n-1}{2}}\right)\right)^{2},\right. \\
& \left.\left(-v_{1},-v_{2}, A u_{1}+\delta P u_{2}+B v_{1}, A u_{2}+P^{*} u_{1}\right) \in\left(\mathcal{D}\left(A^{\frac{n}{2}}\right)\right)^{2} \times\left(\mathcal{D}\left(A^{\frac{n-1}{2}}\right)\right)^{2}\right\},
\end{aligned}
$$

when using the induction assumption $\mathcal{D}\left(\mathcal{A}_{P, \delta}^{n-1}\right)=\mathcal{H}_{n-1}$. Now using assumption (2.12) for $k=n-1$, we see that having

is equivalent to having

$$
\left\{\begin{array}{l}
v_{1} \in \mathcal{D}\left(A^{\frac{n}{2}}\right) ; \quad v_{2} \in \mathcal{D}\left(A^{\frac{n}{2}}\right) \\
A u_{1}+\delta P u_{2}+B v_{1} \in \mathcal{D}\left(A^{\frac{n-1}{2}}\right) \\
A u_{2}+P^{*} u_{1} \in \mathcal{D}\left(A^{\frac{n-1}{2}}\right)
\end{array}\right.
$$

$$
v_{1} \in \mathcal{D}\left(A^{\frac{n}{2}}\right) ; \quad v_{2} \in \mathcal{D}\left(A^{\frac{n}{2}}\right) ; \quad A u_{1} \in \mathcal{D}\left(A^{\frac{n-1}{2}}\right) ; \quad A u_{2} \in \mathcal{D}\left(A^{\frac{n-1}{2}}\right),
$$

that is exactly $\left(u_{1}, u_{2}, v_{1}, v_{2}\right) \in\left(\mathcal{D}\left(A^{\frac{n+1}{2}}\right)\right)^{2} \times\left(\mathcal{D}\left(A^{\frac{n}{2}}\right)\right)^{2}=\mathcal{H}_{n}$. This gives $\mathcal{D}\left(\mathcal{A}_{P, \delta}^{n}\right)=\mathcal{H}_{n}$ and concludes the proof of the lemma. 


\subsubsection{The case $B$ unbounded}

Here, we replace assumption $(A 4 b)$ by the following

$$
(A 4 u)\left\{\begin{array}{c}
\left\langle B u_{1}, i(\phi)\right\rangle_{V_{1}^{\prime}, V_{1}}=0 \quad \forall \phi \in V_{2}, u_{1} \in V_{1}, \text { and } \\
\exists \beta>0,\left\|u_{1}-\Pi_{V} u_{1}\right\|_{H}^{2} \leq \beta\left\langle B u_{1}, u_{1}\right\rangle_{V_{1}^{\prime}, V_{1}} \quad \forall u_{1} \in V_{1} .
\end{array}\right.
$$

Assumption ( $A 4 u$ ) implies that $B$ satisfies a "weak" coercivity property (since the norm on the left hand side of the second inequality in $(A 4 u)$ is the weaker $H$-norm) in the subspace orthogonal to the closed subspace $V_{2}$. As will be seen in Section 4, this property is satisfied for all the systems under view (e.g. wave, plate...). We have the analogous of Theorem 2.4.

Theorem 2.7. Suppose $(A 1),(A 2),(A 3),(A 4 u)$. Then there exists $p_{*} \in\left(0, \lambda_{2}\right]$ such that for all $0<p^{+}<p_{*}$ there exists $\delta_{*}=\delta_{*}\left(p^{+}, p^{-}\right) \in\left(0, \frac{\lambda_{1}}{p^{+}}\right]$, such that for all $\delta \in\left(0, \delta_{*}\right)$, the solution $U(t)=\exp \left(-\mathcal{A}_{P, \delta} t\right) U^{0}$ of $(1.5)$ satisfies for some $C_{n}>0$,

$$
E(U(t)) \leq \frac{C_{n}}{t^{n}} \sum_{i=0}^{2 n} E\left(U^{(i)}(0)\right) \quad \forall t>0, U^{0} \in \mathcal{D}\left(\mathcal{A}_{P, \delta}^{2 n}\right) .
$$

Besides, if $U^{0} \in \mathcal{H}$, then $E(U(t))$ converges to zero as $t$ goes to infinity.

\section{Remark 2.8.}

- As in Theorem 2.4, the constants $p_{*}$ and $\delta_{*}$ here are explicit, that is $p_{*}=\min \left\{\frac{1}{2 \gamma_{2}}, \lambda_{2}\right\}$ and $\delta_{*}=$ $\delta_{*}\left(p^{+}, p^{-}\right)=\min \left\{\frac{\lambda_{1}}{2 \beta_{2}\left(p^{+}\right)^{2} p^{-}}, \frac{\lambda_{2} p^{-}}{2 \beta_{1}\left(p^{+}\right)^{2}}, \frac{\lambda_{1}}{p^{+}}\right\}$.

- Note the difference between the conclusions of Theorems 2.4 and 2.7. For $U^{0} \in D\left(\mathcal{A}_{P, \delta}^{2 n}\right)$, Theorem 2.4 gives a decay rate of the form $C / t^{2 n}$, whereas Theorem 2.7 only gives a decay rate of the form $C / t^{n}$. This comes from the unbounded nature of the operator $B$ (in the applications below, the boundary stabilization).

- Note also that item $($ ii $)$ of Theorem 2.4 has no counterpart here since $P$ and $B$ are not of the same nature.

\section{Proof of the main Results, Theorems 2.4 And 2.7}

\subsection{The stability lemma}

In the sequel, to prove polynomial stability, we shall use the following lemma, which proof can be found in $[1-3]$.

Lemma 3.1. Let $U(t)=\exp (-t \mathcal{A}) U(0)$ a strongly continuous semigroup generated by $(\mathcal{A}, \mathcal{D}(\mathcal{A}))$. Suppose that $t \mapsto E(U(t))$ is a nonincreasing, locally absolutely continuous function from $[0,+\infty)$ to $[0,+\infty)$. Assume moreover that there exists $k \in \mathbb{N}^{*}$ and $c_{i}>0, i=0, \ldots, k$ such that

$$
\int_{S}^{T} E(U(t)) \mathrm{d} t \leq \sum_{i=0}^{k} c_{i} E\left(U^{(i)}(t)\right) \quad \forall 0 \leq S \leq T, \quad \forall U(0) \in \mathcal{D}\left(\mathcal{A}^{k}\right) .
$$

Then, for every $n \in \mathbb{N}$, there exists $C_{n}>0$ such that

$$
E(U(t)) \mathrm{d} t \leq \frac{C_{n}}{t^{n}} \sum_{i=0}^{k n} E\left(U^{(i)}(t)\right) \quad \forall t>0, \quad \forall U(0) \in \mathcal{D}\left(\mathcal{A}^{k n}\right) .
$$

Besides, if $U(0) \in \mathcal{H}$, then $E(U(t))$ converges to zero as $t$ goes to infinity. 
To prove the stability results Theorems 2.4 and 2.7 , we only have to perform energy estimates of the type (3.1). For this we shall use the dissipation relation (2.8), that yields, for all $0 \leq S \leq T$,

$$
\int_{S}^{T}\left\langle B u_{1}^{\prime}, u_{1}^{\prime}\right\rangle_{V^{\prime}, V} \mathrm{~d} t \leq E(U(S))-E(U(T)) \leq E(U(S))
$$

\subsection{Proof of Theorem 2.4, the case $B$ bounded}

The link between $u_{1}$ and $u_{2}$ in the following estimates is given by the following coupling relation.

Lemma 3.2. Assume $(A 4 b)$ and (2.9). Then, for all $U^{0}=\left(u_{1}^{0}, u_{2}^{0}, u_{1}^{1}, u_{2}^{1}\right) \in \mathcal{H}$, the solution $U(t)=\exp \left(-t \mathcal{A}_{P, \delta}\right)$ $U^{0}=\left(u_{1}, u_{2}, v_{1}, v_{2}\right)$ of (1.5) satisfies for some $C=C\left(\delta, p^{+}\right)>0$

$$
\delta \int_{S}^{T}\left(P u_{2}, u_{2}\right)_{H} \mathrm{~d} t \leq \int_{S}^{T}\left(P u_{1}, u_{1}\right)_{H} \mathrm{~d} t+\varepsilon \int_{S}^{T}\left(B u_{2}, u_{2}\right)_{H} \mathrm{~d} t+C\left(1+\frac{1}{\varepsilon}\right) E(U(S))
$$

for all $\varepsilon>0$ and $0 \leq S \leq T$.

Proof. Assume first that $U^{0} \in \mathcal{D}\left(\mathcal{A}_{P, \delta}\right)$. In this case, the solution $U(t)=\exp \left(-t \mathcal{A}_{P, \delta}\right) U^{0}=\left(u_{1}, u_{2}, v_{1}, v_{2}\right)$ of $(1.5)$ is in $\mathcal{C}^{0}\left([0,+\infty) ; \mathcal{D}\left(\mathcal{A}_{P, \delta}\right) \cap \mathcal{C}^{1}([0,+\infty) ; \mathcal{H})\right.$. Hence $U=\left(u_{1}, u_{2}, v_{1}, v_{2}\right)$ satisfies

$$
\begin{cases}v_{1}=u_{1}^{\prime}, \quad v_{2}=u_{2}^{\prime}, & \\ u_{1}^{\prime \prime}+A u_{1}+B u_{1}^{\prime}+\delta P u_{2}=0 & \text { in } H, \\ u_{2}^{\prime \prime}+A u_{2}+P^{*} u_{1}=0 & \text { in } H .\end{cases}
$$

As a consequence, we have $\forall 0 \leq S \leq T$,

$$
\int_{S}^{T}\left(u_{1}^{\prime \prime}+A u_{1}+B u_{1}^{\prime}+\delta P u_{2}, u_{2}\right)_{H}-\left(u_{2}^{\prime \prime}+A u_{2}+P^{*} u_{1}, u_{1}\right)_{H} \mathrm{~d} t=0 .
$$

We first notice that $\left(A u_{1}, u_{2}\right)_{H}-\left(A u_{2}, u_{1}\right)_{H}=0$ since $A$ is selfadjoint, and

$$
\begin{aligned}
\left|\int_{S}^{T}\left(u_{1}^{\prime \prime}, u_{2}\right)_{H}-\left(u_{2}^{\prime \prime}, u_{1}\right)_{H} \mathrm{~d} t\right| & =\left|\left[\left(u_{1}^{\prime}, u_{2}\right)_{H}-\left(u_{2}^{\prime}, u_{1}\right)_{H}\right]_{S}^{T}\right| \\
& \leq \frac{1}{2} \sum_{j=1,2}\left(\left\|u_{j}^{\prime}(S)\right\|_{H}^{2}+\left\|u_{j}^{\prime}(T)\right\|_{H}^{2}+\left\|u_{j}(S)\right\|_{H}^{2}+\left\|u_{j}(T)\right\|_{H}^{2}\right) .
\end{aligned}
$$

From (2.6) and (2.9), each of the terms here is bounded by the energy, i.e., for $j=1,2$,

$$
\left\|u_{j}^{\prime}(S)\right\|_{H}^{2}+\left\|u_{j}(S)\right\|_{H}^{2} \leq C E(U(S)) \quad \text { and } \quad\left\|u_{j}^{\prime}(T)\right\|_{H}^{2}+\left\|u_{j}(T)\right\|_{H}^{2} \leq C E(U(T)),
$$

where $C=C\left(\delta, p^{+}\right)$. Since the energy is decaying and $T \geq S$, we have $E(U(T)) \leq E(U(S))$, so that

$$
\left|\int_{S}^{T}\left(u_{1}^{\prime \prime}, u_{2}\right)_{H}-\left(u_{2}^{\prime \prime}, u_{1}\right)_{H} \mathrm{~d} t\right| \leq C E(U(S)) .
$$

Now, (3.4) becomes

$$
\begin{aligned}
\delta \int_{S}^{T}\left(P u_{2}, u_{2}\right)_{H} \mathrm{~d} t \leq \int_{S}^{T}\left(P u_{1}, u_{1}\right)_{H} \mathrm{~d} t+\left|\int_{S}^{T}\left(B u_{1}^{\prime}, u_{2}\right)_{H} \mathrm{~d} t\right|+C E(U(S)) \\
\leq \int_{S}^{T}\left(P u_{1}, u_{1}\right)_{H} \mathrm{~d} t+\frac{C}{\varepsilon} \int_{S}^{T}\left(B u_{1}^{\prime}, u_{1}^{\prime}\right)_{H} \mathrm{~d} t+\varepsilon \int_{S}^{T}\left(B u_{2}, u_{2}\right)_{H} \mathrm{~d} t+C E(U(S)),
\end{aligned}
$$


for all $\varepsilon>0$, since $B$ is selfadjoint on $H$. From (3.2), we have $\int_{S}^{T}\left(B u_{1}^{\prime}, u_{1}^{\prime}\right)_{H} \mathrm{~d} t \leq C E(U(S))$, and (3.5) yields

$$
\delta \int_{S}^{T}\left(P u_{2}, u_{2}\right)_{H} \mathrm{~d} t \leq \int_{S}^{T}\left(P u_{1}, u_{1}\right)_{H} \mathrm{~d} t+\varepsilon \int_{S}^{T}\left(B u_{2}, u_{2}\right)_{H} \mathrm{~d} t+C\left(1+\frac{1}{\varepsilon}\right) E(U(S))
$$

for all $\varepsilon>0$ and $0 \leq S \leq T$. By a density argument, we deduce that (3.6) holds for every $U^{0} \in \mathcal{H}$.

We can now prove Theorem 2.4.

Proof of Theorem 2.4. We first prove assertion (i). Assume that $U^{0} \in \mathcal{D}\left(\mathcal{A}_{P, \delta}\right)$, then, the solution $U$ of (1.5) is in $\mathcal{C}^{0}\left([0,+\infty) ; \mathcal{D}\left(\mathcal{A}_{P, \delta}\right)\right) \cap \mathcal{C}^{1}\left([0,+\infty) ; \mathcal{H}\right.$ ) (see Prop. 2.2). We denote by $e_{j}(t)=1 / 2\left(\left\|u_{j}^{\prime}\right\|_{H}^{2}+\left\|u_{j}\right\|_{V}^{2}\right), j=1,2$, the partial energies. The regularity of $U(t)$ gives in particular $P u_{2} \in \mathcal{C}^{1}([0,+\infty) ; H)$ and $P^{*} u_{1} \in \mathcal{C}^{1}([0,+\infty) ; H)$, so that assumptions $(A 2)$ and $(A 3)$ yield

$$
\begin{gathered}
\int_{S}^{T} e_{1}(t) \mathrm{d} t \leq C E(U(S))+\beta_{1} \int_{S}^{T}\left\|\delta P u_{2}(t)\right\|_{H}^{2} \mathrm{~d} t+\gamma_{1} \int_{S}^{T}\left\langle B u_{1}^{\prime}, u_{1}^{\prime}\right\rangle_{V^{\prime}, V} \mathrm{~d} t, \\
\int_{S}^{T} e_{2}(t) \mathrm{d} t \leq C E(U(S))+\beta_{2} \int_{S}^{T}\left\|P^{*} u_{1}(t)\right\|_{H}^{2} \mathrm{~d} t+\gamma_{2} \int_{S}^{T}\left\|\Pi_{P} u_{2}^{\prime}(t)\right\|_{H}^{2} \mathrm{~d} t,
\end{gathered}
$$

since $e_{j}(t) \leq C E(U(t)) \leq C E(U(S))$ for $t \geq S$. From (3.2), we have $\int_{S}^{T}\left\langle B u_{1}^{\prime}, u_{1}^{\prime}\right\rangle_{V^{\prime}, V} \mathrm{~d} t \leq E(U(S))$, so that (3.7) yields

$$
\int_{S}^{T} e_{1}(t) \mathrm{d} t \leq C E(U(S))+\beta_{1} \delta^{2}\left(p^{+}\right)^{2} \int_{S}^{T}\left\|u_{2}(t)\right\|_{H}^{2} \mathrm{~d} t .
$$

On the other side, assumption $(A 1)$ and the coupling relation (3.3) of Lemma 3.2, applied to $U^{\prime} \in \mathcal{C}^{0}([0,+\infty) ; \mathcal{H})$ give, for all $\varepsilon>0$,

$$
\delta p^{-} \int_{S}^{T}\left\|\Pi_{P} u_{2}^{\prime}\right\|_{H}^{2} \mathrm{~d} t \leq \delta \int_{S}^{T}\left(P u_{2}^{\prime}, u_{2}^{\prime}\right)_{H} \mathrm{~d} t \leq p^{+} \int_{S}^{T}\left\|u_{1}^{\prime}\right\|_{H}^{2} \mathrm{~d} t+\varepsilon b^{+} \int_{S}^{T}\left\|u_{2}^{\prime}\right\|_{H}^{2} \mathrm{~d} t+C_{\varepsilon} E\left(U^{\prime}(S)\right) .
$$

Replacing (3.10) in (3.8), we obtain

$$
\begin{aligned}
\delta p^{-} \int_{S}^{T} e_{2}(t) \mathrm{d} t \leq C E(U(S))+\beta_{2} \delta p^{-}\left(p^{+}\right)^{2} & \int_{S}^{T}\left\|u_{1}\right\|_{H}^{2} \mathrm{~d} t \\
& +\gamma_{2} p^{+} \int_{S}^{T}\left\|u_{1}^{\prime}\right\|_{H}^{2} \mathrm{~d} t+\varepsilon \gamma_{2} b^{+} \int_{S}^{T}\left\|u_{2}^{\prime}\right\|_{H}^{2} \mathrm{~d} t+C_{\varepsilon} E\left(U^{\prime}(S)\right) .
\end{aligned}
$$

Then, recalling that for all $v \in V,\|v\|_{H}^{2} \leq 1 / \lambda\|v\|_{V}^{2}$, and adding (3.9) and (3.11), we obtain, for all $\varepsilon>0$,

$$
\begin{aligned}
\left(\frac{1}{2}-\gamma_{2} p^{+}\right) \int_{S}^{T}\left\|u_{1}^{\prime}\right\|_{H}^{2} \mathrm{~d} t+\left(\frac{1}{2}-\frac{\beta_{2} \delta\left(p^{+}\right)^{2} p^{-}}{\lambda}\right) \int_{S}^{T}\left\|u_{1}\right\|_{V}^{2} \mathrm{~d} t+\left(\frac{\delta p^{-}}{2}-\varepsilon \gamma_{2} b^{+}\right) \int_{S}^{T}\left\|u_{2}^{\prime}\right\|_{H}^{2} \mathrm{~d} t \\
+\left(\frac{\delta p^{-}}{2}-\frac{\beta_{1} \delta^{2}\left(p^{+}\right)^{2}}{\lambda}\right) \int_{S}^{T}\left\|u_{2}\right\|_{V}^{2} \mathrm{~d} t \leq C E(U(S))+C_{\varepsilon} E\left(U^{\prime}(S)\right) .
\end{aligned}
$$


We now set $p_{*}=\min \left\{\frac{1}{2 \gamma_{2}}, \lambda\right\}>0$ and $\delta_{*}=\delta_{*}\left(p^{+}, p^{-}\right)=\min \left\{\frac{\lambda}{2 \beta_{2}\left(p^{+}\right)^{2} p^{-}}, \frac{\lambda p^{-}}{2 \beta_{1}\left(p^{+}\right)^{2}}, \frac{\lambda}{p^{+}}\right\}>0$. Then for all $p^{+} \in\left(0, p_{*}\right)$ and $\delta \in\left(0, \delta_{*}\right)$, one can choose $0<\varepsilon<\frac{\delta p^{-}}{2 b^{+}}$, so that the following bound on the energy holds

$$
\begin{aligned}
\int_{S}^{T} E(U(t)) \mathrm{d} t & \leq C\left(\delta, p^{+}\right) \int_{S}^{T}\left(\left\|u_{1}^{\prime}(t)\right\|_{H}^{2}+\left\|u_{1}(t)\right\|_{V}^{2} \mathrm{~d} t+\left\|u_{2}^{\prime}(t)\right\|_{H}^{2}+\left\|u_{2}(t)\right\|_{V}^{2}\right) \mathrm{d} t \\
& \leq C\left(\delta, p^{+}, p^{-}\right)\left(E(U(S))+E\left(U^{\prime}(S)\right)\right), \quad \forall 0 \leq S \leq T, \quad \forall U^{0} \in \mathcal{D}\left(\mathcal{A}_{P, \delta}\right),
\end{aligned}
$$

from (3.12) and the choice of $p_{*}$ and $\delta_{*}$. Using now Lemma 3.1, we obtain

$$
E(U(t)) \leq \frac{C_{n}}{t^{n}} \sum_{i=0}^{n} E\left(U^{(i)}(0)\right) \quad \forall t>0, \quad \forall U^{0} \in \mathcal{D}\left(\mathcal{A}_{P, \delta}^{n}\right),
$$

and $(i)$ is proved.

We now prove the first case of $(i i)$ and suppose (2.10). Thanks to this assumption, the coupling relation (3.3) of Lemma 3.2, applied to $U \in \mathcal{C}^{1}([0,+\infty) ; \mathcal{H})$ gives for all $\varepsilon>0$

$$
\delta \int_{S}^{T}\left(P u_{2}, u_{2}\right)_{H} \mathrm{~d} t \leq \int_{S}^{T}\left(P u_{1}, u_{1}\right)_{H} \mathrm{~d} t+\frac{\varepsilon b^{+}}{p^{-}} \int_{S}^{T}\left(P u_{2}, u_{2}\right)_{H} \mathrm{~d} t+C_{\varepsilon} E(U(S)) .
$$

This and assumption (2.10) yield for all $0<\varepsilon<\frac{p^{-} \delta}{b^{+}}$

$$
\int_{S}^{T}\left\|P u_{2}\right\|_{H}^{2} \mathrm{~d} t \leq p^{+} \int_{S}^{T}\left(P u_{2}, u_{2}\right)_{H} \mathrm{~d} t \leq \frac{\left(p^{+}\right)^{2}}{\delta-\varepsilon b^{+} / p^{-}} \int_{S}^{T}\left\|u_{1}\right\|_{H}^{2} \mathrm{~d} t+C_{\varepsilon} E(U(S))
$$

so that (3.7) gives, for all $0<\varepsilon<\frac{p^{-} \delta}{b^{+}}$,

$$
\int_{S}^{T} e_{1}(t) \mathrm{d} t \leq \frac{\beta_{1} \delta^{2}\left(p^{+}\right)^{2}}{\delta-\varepsilon b^{+} / p^{-}} \int_{S}^{T}\left\|u_{1}\right\|_{H}^{2} \mathrm{~d} t+C_{\varepsilon} E(U(S)) .
$$

Now we set $\varepsilon=(1-\eta) \frac{p^{-} \delta}{b^{+}}$and we obtain, for all $\eta \in(0,1)$,

$$
\frac{1}{2} \int_{S}^{T}\left\|u_{1}^{\prime}\right\|_{H}^{2} \mathrm{~d} t+\left(\frac{1}{2}-\frac{\beta_{1} \delta\left(p^{+}\right)^{2}}{\eta \lambda}\right) \int_{S}^{T}\left\|u_{1}\right\|_{V}^{2} \mathrm{~d} t \leq C_{\eta} E(U(S)) .
$$

Choosing $p_{*}=\min \left\{\frac{1}{2 \beta_{1}}, \lambda\right\}$, we have for every $p^{+}<p_{*}$,

$$
\left(p^{+}\right)^{2}<\frac{\lambda}{2 \beta_{1} \delta}
$$

since $\delta$ is chosen such that $0<p^{+}<\lambda / \delta$. From (3.14), for all $p^{+} \in\left(0, p_{*}\right)$ and $\delta \in\left(0, \lambda / p^{+}\right)$, there exists $0<\eta<1$, such that $\frac{2 \beta_{1} \delta\left(p^{+}\right)^{2}}{\lambda}<\eta$ and (3.13) implies the existence of $C=C\left(\delta, p^{+}\right)$, such that

$$
\int_{S}^{T} e_{1}(t) \mathrm{d} t \leq C E(U(S))
$$

Besides, the coupling relation (3.3) of Lemma 3.2, applied to $U^{\prime} \in \mathcal{C}^{0}([0,+\infty) ; \mathcal{H})$ implies in this case, for all $\varepsilon>0$,

$$
\delta \int_{S}^{T}\left(P u_{2}^{\prime}, u_{2}^{\prime}\right)_{H} \mathrm{~d} t \leq \int_{S}^{T}\left(P u_{1}^{\prime}, u_{1}^{\prime}\right)_{H} \mathrm{~d} t+\frac{\varepsilon b^{+}}{p^{-}} \int_{S}^{T}\left(P u_{2}^{\prime}, u_{2}^{\prime}\right)_{H} \mathrm{~d} t+C_{\varepsilon} E\left(U^{\prime}(S)\right) .
$$


Proceeding as above, we obtain for all $0<\varepsilon<p^{-} \delta / b^{+}$,

$$
p^{-}\left(\delta-\frac{\varepsilon b^{+}}{p^{-}}\right) \int_{S}^{T}\left\|\Pi_{P} u_{2}^{\prime}\right\|_{H}^{2} \mathrm{~d} t \leq p^{+} \int_{S}^{T}\left\|u_{1}^{\prime}\right\|_{H}^{2} \mathrm{~d} t+C_{\varepsilon} E\left(U^{\prime}(S)\right) .
$$

Fixing $\varepsilon \in\left(0, p^{-} \delta / b^{+}\right)$and replacing (3.16) in (3.8), we obtain for some $C=C\left(\delta, b^{+}, p^{-}, p^{+}\right)$,

$$
\begin{aligned}
\int_{S}^{T} e_{2}(t) \mathrm{d} t & \leq C E(U(S))+\beta_{2}\left(p^{+}\right)^{2} \int_{S}^{T}\left\|u_{1}\right\|_{H}^{2} \mathrm{~d} t+C \int_{S}^{T}\left\|u_{1}^{\prime}\right\|_{H}^{2} \mathrm{~d} t+C E\left(U^{\prime}(S)\right) \\
& \leq C \int_{S}^{T} e_{1}(t) \mathrm{d} t+C E(U(S))+C E\left(U^{\prime}(S)\right) .
\end{aligned}
$$

Estimate (3.15) on $e_{1}$ gives

$$
\int_{S}^{T} e_{2}(t) \leq C E(U(S))+C E\left(U^{\prime}(S)\right)
$$

so that the following bound on the energy holds

$$
\begin{aligned}
\int_{S}^{T} E(U(t)) \mathrm{d} t & \leq C\left(\delta, p^{+}\right) \int_{S}^{T}\left(\left\|u_{1}^{\prime}(t)\right\|_{H}^{2}+\left\|u_{1}(t)\right\|_{V}^{2} \mathrm{~d} t+\left\|u_{2}^{\prime}(t)\right\|_{H}^{2}+\left\|u_{2}(t)\right\|_{V}^{2}\right) \mathrm{d} t \\
& \leq C\left(\delta, p^{+}, p^{-}\right)\left(E(U(S))+E\left(U^{\prime}(S)\right)\right), \quad \forall 0 \leq S \leq T, \quad \forall U^{0} \in \mathcal{D}\left(\mathcal{A}_{P, \delta}\right) .
\end{aligned}
$$

We conclude the proof of the first part of (ii) as in case (i) with Lemma 3.1.

To conclude the proof of $(i i)$, suppose now assumption (2.11), i.e., there exists $b^{-}>0$ such that $(P v, v)_{H} \leq$ $p^{+} / b^{-}(B v, v)_{H}$, for all $v \in H$. In this case, the coupling relation (3.3) of Lemma 3.2, applied to $U^{\prime} \in$ $\mathcal{C}^{0}([0,+\infty) ; \mathcal{H})$ gives

$$
\delta p^{-} \int_{S}^{T}\left\|\Pi_{P} u_{2}^{\prime}\right\|_{H}^{2} \mathrm{~d} t \leq \delta \int_{S}^{T}\left(P u_{2}^{\prime}, u_{2}^{\prime}\right)_{H} \mathrm{~d} t \leq \frac{p^{+}}{b^{-}} \int_{S}^{T}\left(B u_{1}^{\prime}, u_{1}^{\prime}\right)_{H} \mathrm{~d} t+\varepsilon b^{+} \int_{S}^{T}\left\|u_{2}^{\prime}\right\|_{H}^{2} \mathrm{~d} t+C_{\varepsilon} E\left(U^{\prime}(S)\right) .
$$

We recall that $\int_{S}^{T}\left(B u_{1}^{\prime}, u_{1}^{\prime}\right)_{H} \mathrm{~d} t \leq E(U(S))$. Replacing (3.17) in (3.8), we obtain

$$
\delta \int_{S}^{T} e_{2}(t) \mathrm{d} t \leq C E(U(S))+\beta_{2} \delta\left(p^{+}\right)^{2} \int_{S}^{T}\left\|u_{1}\right\|_{H}^{2} \mathrm{~d} t+\frac{\varepsilon b^{+} \gamma_{2}}{p^{-}} \int_{S}^{T}\left\|u_{2}^{\prime}\right\|_{H}^{2} \mathrm{~d} t+C_{\varepsilon} E\left(U^{\prime}(S)\right),
$$

which, summed with (3.9), yields

$$
\begin{aligned}
\frac{1}{2} \int_{S}^{T}\left\|u_{1}^{\prime}\right\|_{H}^{2} \mathrm{~d} t+\left(\frac{1}{2}-\frac{\beta_{2} \delta\left(p^{+}\right)^{2}}{\lambda}\right) \int_{S}^{T} & \left\|u_{1}\right\|_{V}^{2} \mathrm{~d} t+\left(\frac{\delta}{2}-\frac{\varepsilon b^{+} \gamma_{2}}{p^{-}}\right) \int_{S}^{T}\left\|u_{2}^{\prime}\right\|_{H}^{2} \mathrm{~d} t \\
& +\left(\frac{\delta}{2}-\frac{\beta_{1} \delta^{2}\left(p^{+}\right)^{2}}{\lambda}\right) \int_{S}^{T}\left\|u_{2}\right\|_{V}^{2} \mathrm{~d} t \leq C E(U(S))+C_{\varepsilon} E\left(U^{\prime}(S)\right) .
\end{aligned}
$$

Now, setting $p_{*}=\min \left\{\frac{1}{2 \beta_{1}}, \frac{1}{2 \beta_{2}}, \lambda\right\}$, we have for every $p^{+}<p_{*}$

$$
\left(p^{+}\right)^{2}<\min \left\{\frac{\lambda}{2 \delta \beta_{1}} ; \frac{\lambda}{2 \delta \beta_{2}}\right\}
$$


since $\delta$ is chosen such that $0<p^{+}<\lambda / \delta$. From (3.20), for all $p^{+} \in\left(0, p_{*}\right)$ and $\delta \in\left(0, \lambda / p^{+}\right)$, one can choose $0<\varepsilon<\frac{\delta p^{-}}{2 b^{+} \gamma_{2}}$, so that the following bound on the energy holds

$$
\begin{aligned}
\int_{S}^{T} E(U(t)) \mathrm{d} t & \leq C\left(\delta, p^{+}\right) \int_{S}^{T}\left(\left\|u_{1}^{\prime}(t)\right\|_{H}^{2}+\left\|u_{1}(t)\right\|_{V}^{2} \mathrm{~d} t+\left\|u_{2}^{\prime}(t)\right\|_{H}^{2}+\left\|u_{2}(t)\right\|_{V}^{2}\right) \mathrm{d} t \\
& \leq C\left(\delta, p^{+}, p^{-}\right)\left(E(U(S))+E\left(U^{\prime}(S)\right)\right), \quad \forall 0 \leq S \leq T, \quad \forall U^{0} \in \mathcal{D}\left(\mathcal{A}_{P, \delta}\right) .
\end{aligned}
$$

We conclude the proof of the last part of $(i i)$ as before with Lemma 3.1. This ends the proof of Theorem 2.4

\subsection{Proof of Theorem 2.7, the case $B$ unbounded}

We first state the analogous of Lemma 3.2, that provides a coupling relation between $u_{1}$ and $u_{2}$.

Lemma 3.3. Assume $(A 4 u)$ and (2.7). Then, for all $U^{0}=\left(u_{1}^{0}, u_{2}^{0}, u_{1}^{1}, u_{2}^{1}\right) \in \mathcal{D}\left(\mathcal{A}_{P, \delta}\right)$, the solution $U(t)=$ $\exp \left(-t \mathcal{A}_{P, \delta}\right) U^{0}=\left(u_{1}, u_{2}, v_{1}, v_{2}\right)$ of (1.5) satisfies for some $C=C\left(\delta, p^{+}\right)>0$

$$
\delta \int_{S}^{T}\left(P u_{2}, u_{2}\right)_{H} \mathrm{~d} t \leq \int_{S}^{T}\left(P^{*} u_{1}, \Pi_{V} u_{1}\right)_{H} \mathrm{~d} t+\varepsilon \int_{S}^{T}\left\|u_{2}\right\|_{H}^{2} \mathrm{~d} t+\frac{C}{\varepsilon} E\left(U^{\prime}(S)\right)+C E(U(S))
$$

for all $\varepsilon>0$ and $0 \leq S \leq T$.

Remark 3.4. The main difference with the bounded case (Lem. 3.2) is that here, the energy of the derivative of $U$ is needed in the coupling relation.

Proof. Assume first that $U^{0} \in \mathcal{D}\left(\mathcal{A}_{P . \delta}^{2}\right)$. In this case, the solution $U(t)=\exp \left(-t \mathcal{A}_{P, \delta}\right) U^{0}=\left(u_{1}, u_{2}, v_{1}, v_{2}\right)$ of $(1.5)$ is in $\mathcal{C}^{0}\left([0,+\infty) ; \mathcal{D}\left(\mathcal{A}_{P, \delta}^{2}\right)\right) \cap \mathcal{C}^{1}\left([0,+\infty) ; \mathcal{D}\left(\mathcal{A}_{P, \delta}\right)\right) \cap \mathcal{C}^{2}([0,+\infty) ; \mathcal{H})$. Hence $U=\left(u_{1}, u_{2}, v_{1}, v_{2}\right)$ satisfies

$$
\begin{cases}v_{1}=u_{1}^{\prime}, \quad v_{2}=u_{2}^{\prime}, & \\ u_{1}^{\prime \prime}+A_{1} u_{1}+B u_{1}^{\prime}+\delta P u_{2}=0 & \text { in } H, \\ u_{2}^{\prime \prime}+A_{2} u_{2}+P^{*} u_{1}=0 & \text { in } H .\end{cases}
$$

As a consequence, we have $\forall 0 \leq S \leq T$,

$$
\int_{S}^{T}\left(u_{1}^{\prime \prime}+A_{1} u_{1}+B u_{1}^{\prime}+\delta P u_{2}, u_{2}\right)_{H}-\left(u_{2}^{\prime \prime}+A_{2} u_{2}+P^{*} u_{1}, \Pi_{V} u_{1}\right)_{H} \mathrm{~d} t=0
$$

i.e., $K_{1}+K_{2}+K_{3}=0$, with

$$
\begin{aligned}
K_{1} & =\int_{S}^{T}\left(u_{1}^{\prime \prime}, u_{2}\right)_{H}-\left(u_{2}^{\prime \prime}, \Pi_{V} u_{1}\right)_{H} \mathrm{~d} t \\
K_{2} & =\int_{S}^{T}\left(A_{1} u_{1}+B u_{1}^{\prime}, u_{2}\right)_{H}-\left(A_{2} u_{2}, \Pi_{V} u_{1}\right)_{H} \mathrm{~d} t \\
K_{3} & =\int_{S}^{T} \delta\left(P u_{2}, u_{2}\right)_{H}-\left(P^{*} u_{1}, \Pi_{V} u_{1}\right)_{H} \mathrm{~d} t .
\end{aligned}
$$

We first consider $K_{1}$. Since $U^{0}$ is taken in $\mathcal{D}\left(\mathcal{A}_{P, \delta}^{2}\right), u_{i} \in \mathcal{C}^{2}\left([0,+\infty) ; V_{i}\right)$ for $i=1,2$. Hence, $\left(\Pi_{V} u_{1}\right)^{\prime \prime}=$ $\Pi_{V}\left(u_{1}^{\prime \prime}\right)$ and

$$
K_{1}=\int_{S}^{T}\left(u_{1}^{\prime \prime}-\Pi_{V} u_{1}^{\prime \prime}, u_{2}\right)_{H} \mathrm{~d} t+\left[\left(\Pi_{V} u_{1}^{\prime}, u_{2}\right)_{H}-\left(\Pi_{V} u_{1}, u_{2}^{\prime}\right)_{H}\right]_{S}^{T} .
$$


As a consequence, for all $\varepsilon>0$,

$$
\left|K_{1}\right| \leq \frac{C}{\varepsilon} \int_{S}^{T}\left\|u_{1}^{\prime \prime}-\Pi_{V} u_{1}^{\prime \prime}\right\|_{H}^{2}+\varepsilon \int_{S}^{T}\left\|u_{2}\right\|_{H}^{2} \mathrm{~d} t+\sum_{j=1,2}\left(\left\|u_{j}^{\prime}(S)\right\|_{H}^{2}+\left\|u_{j}^{\prime}(T)\right\|_{H}^{2}+\left\|u_{j}(S)\right\|_{H}^{2}+\left\|u_{j}(T)\right\|_{H}^{2}\right) .
$$

From (2.6) and (2.7), each of the terms of the sum is bounded by the energy, i.e., for $j=1,2$,

$$
\left\|u_{j}^{\prime}(S)\right\|_{H}^{2}+\left\|u_{j}(S)\right\|_{H}^{2} \leq C E(U(S)) \quad \text { and } \quad\left\|u_{j}^{\prime}(T)\right\|_{H}^{2}+\left\|u_{j}(T)\right\|_{H}^{2} \leq C E(U(T)) \leq C E(U(S)),
$$

since the energy is decaying and $T \geq S$. Replacing this in (3.22), and using assumption $(A 4 u)$, we obtain, for all $\varepsilon>0$,

$$
\left|K_{1}\right| \leq \frac{C}{\varepsilon} \int_{S}^{T} \beta\left\langle B u_{1}^{\prime \prime}, u_{1}^{\prime \prime}\right\rangle_{V_{1}^{\prime}, V_{1}} \mathrm{~d} t+\varepsilon \int_{S}^{T}\left\|u_{2}\right\|_{H}^{2} \mathrm{~d} t+C E(U(S)) .
$$

On the other side, Proposition 2.2 gives $E^{\prime}(U(t))=-\left\langle B u_{1}^{\prime}, u_{1}^{\prime}\right\rangle_{V_{1}^{\prime}, V_{1}}$ for $U^{0} \in \mathcal{D}\left(\mathcal{A}_{P, \delta}\right)$, so that we have $E^{\prime}\left(U^{\prime}(t)\right)=-\left\langle B u_{1}^{\prime \prime}, u_{1}^{\prime \prime}\right\rangle_{V_{1}^{\prime}, V_{1}}$ for $U^{0} \in \mathcal{D}\left(\mathcal{A}_{P, \delta}^{2}\right)$. Recalling that $E(U(\cdot))$ and $E\left(U^{\prime}(\cdot)\right)$ are nonincreasing, we obtain, for all $\varepsilon>0$,

$$
\left|K_{1}\right| \leq \varepsilon \int_{S}^{T}\left\|u_{2}\right\|_{H}^{2} \mathrm{~d} t+\frac{C}{\varepsilon} E\left(U^{\prime}(S)\right)+C E(U(S))
$$

We now consider $K_{2}$. From Assumption $(A 4 u)$, we have,

$$
\left(A_{1} u_{1}+B u_{1}^{\prime}, u_{2}\right)_{H}=\left\langle A_{1} u_{1}+B u_{1}^{\prime}, i\left(u_{2}\right)\right\rangle_{V_{1}^{\prime}, V_{1}}=\left\langle A_{1} u_{1}, i\left(u_{2}\right)\right\rangle_{V_{1}^{\prime}, V_{1}},
$$

since $U^{0} \in \mathcal{D}\left(\mathcal{A}_{P, \delta}^{2}\right)$ yields $u_{2} \in \mathcal{D}\left(A_{2}\right) \subset V_{2}$. The definition of $\Pi_{V}$ also gives

$$
\left\langle A_{1} u_{1}, i\left(u_{2}\right)\right\rangle_{V_{1}^{\prime}, V_{1}}=\left\langle A_{1} i\left(\Pi_{V} u_{1}\right), i\left(u_{2}\right)\right\rangle_{V_{1}^{\prime}, V_{1}}=\left\langle A_{2} \Pi_{V} u_{1}, u_{2}\right\rangle_{V_{2}^{\prime}, V_{2}}=\left\langle A_{2} u_{2}, \Pi_{V} u_{1}\right\rangle_{V_{2}^{\prime}, V_{2}} .
$$

Moreover, since $u_{2} \in \mathcal{D}\left(A_{2}\right)$, we have $\left\langle A_{2} u_{2}, \Pi_{V} u_{1}\right\rangle_{V_{2}^{\prime}, V_{2}}=\left(A_{2} u_{2}, \Pi_{V} u_{1}\right)_{H}$, so that

$$
\left(A_{1} u_{1}+B u_{1}^{\prime}, u_{2}\right)_{H}=\left(A_{2} u_{2}, \Pi_{V} u_{1}\right)_{H},
$$

and $K_{2}=0$.

Finally, replacing in

$$
\int_{S}^{T} \delta\left(P u_{2}, u_{2}\right)_{H} \mathrm{~d} t=\int_{S}^{T}\left(P^{*} u_{1}, \Pi_{V} u_{1}\right)_{H} \mathrm{~d} t-K_{1}-K_{2}
$$

the estimates on $\left|K_{1}\right|$ and $K_{2}$, we have for all $\varepsilon>0$,

$$
\int_{S}^{T} \delta\left(P u_{2}, u_{2}\right)_{H} \mathrm{~d} t \leq \int_{S}^{T}\left(P^{*} u_{1}, \Pi_{V} u_{1}\right)_{H} \mathrm{~d} t+\varepsilon \int_{S}^{T}\left\|u_{2}\right\|_{H}^{2} \mathrm{~d} t+\frac{C}{\varepsilon} E\left(U^{\prime}(S)\right)+C E(U(S)) .
$$

This concludes the proof of the proposition for an initial datum $U^{0} \in \mathcal{D}\left(\mathcal{A}_{P, \delta}^{2}\right)$. By a density argument, we deduce that (3.23) holds for every $U^{0} \in \mathcal{D}\left(\mathcal{A}_{P, \delta}\right)$.

We can now prove Theorem 2.7. This proof follows the same steps as in the proof of Theorem 2.4 point $(i)$. We give it for the sake of completeness.

Proof of Theorem 2.7. Assume that $U^{0} \in \mathcal{D}\left(\mathcal{A}_{P, \delta}^{2}\right)$, then, the solution $U$ of (1.5) is in

$$
\mathcal{C}^{0}\left([0,+\infty) ; \mathcal{D}\left(\mathcal{A}_{P, \delta}^{2}\right)\right) \cap \mathcal{C}^{1}\left([0,+\infty) ; \mathcal{D}\left(\mathcal{A}_{P, \delta}\right)\right) \cap \mathcal{C}^{2}([0,+\infty) ; \mathcal{H})
$$


(see Prop. 2.2). We recall the notation $e_{j}(t)=1 / 2\left(\left\|u_{j}^{\prime}\right\|_{H}^{2}+\left\|u_{j}\right\|_{V_{j}}^{2}\right), j=1,2$. The regularity of $U(t)$ gives in particular $P u_{2} \in \mathcal{C}^{1}([0,+\infty) ; H)$ and $P^{*} u_{1} \in \mathcal{C}^{1}([0,+\infty) ; H)$, so that Assumptions $(A 2)$ and $(A 3)$ yield

$$
\begin{gathered}
\int_{S}^{T} e_{1}(t) \mathrm{d} t \leq C E(U(S))+\beta_{1} \int_{S}^{T}\left\|\delta P u_{2}(t)\right\|_{H}^{2} \mathrm{~d} t+\gamma_{1} \int_{S}^{T}\left\langle B u_{1}^{\prime}, u_{1}^{\prime}\right\rangle_{V_{1}^{\prime}, V_{1}} \mathrm{~d} t \\
\int_{S}^{T} e_{2}(t) \mathrm{d} t \leq C E(U(S))+\beta_{2} \int_{S}^{T}\left\|P^{*} u_{1}(t)\right\|_{H}^{2} \mathrm{~d} t+\gamma_{2} \int_{S}^{T}\left\|\Pi_{P} u_{2}^{\prime}(t)\right\|_{H}^{2} \mathrm{~d} t .
\end{gathered}
$$

From (3.2), we have $\int_{S}^{T}\left\langle B u_{1}^{\prime}, u_{1}^{\prime}\right\rangle_{V_{1}^{\prime}, V_{1}} \mathrm{~d} t \leq E(U(S))$, so that (3.24) yields

$$
\int_{S}^{T} e_{1}(t) \mathrm{d} t \leq C E(U(S))+\beta_{1} \delta^{2}\left(p^{+}\right)^{2} \int_{S}^{T}\left\|u_{2}(t)\right\|_{H}^{2} \mathrm{~d} t
$$

On the other side, Assumption (A1) and the coupling relation (3.21) of Lemma 3.3, applied to $U^{\prime} \in \mathcal{C}^{0}([0,+\infty)$; $\left.\mathcal{D}\left(\mathcal{A}_{P, \delta}\right)\right)$ give, for all $\varepsilon>0$,

$$
\begin{aligned}
\delta p^{-} \int_{S}^{T}\left\|\Pi_{P} u_{2}^{\prime}\right\|_{H}^{2} \mathrm{~d} t & \leq \delta \int_{S}^{T}\left(P u_{2}^{\prime}, u_{2}^{\prime}\right)_{H} \mathrm{~d} t \\
& \leq p^{+} \int_{S}^{T}\left\|u_{1}^{\prime}\right\|_{H}^{2} \mathrm{~d} t+\varepsilon \int_{S}^{T}\left\|u_{2}^{\prime}\right\|_{H}^{2} \mathrm{~d} t+C_{\varepsilon} E\left(U^{\prime \prime}(S)\right)+C E\left(U^{\prime}(S)\right)
\end{aligned}
$$

Replacing (3.27) in (3.25), we obtain

$$
\begin{aligned}
\delta p^{-} \int_{S}^{T} e_{2}(t) \mathrm{d} t \leq C E(U(S))+C E\left(U^{\prime}(S)\right)+ & \beta_{2} \delta p^{-}\left(p^{+}\right)^{2} \int_{S}^{T}\left\|u_{1}(t)\right\|_{H}^{2} \mathrm{~d} t \\
& +\gamma_{2} p^{+} \int_{S}^{T}\left\|u_{1}^{\prime}\right\|_{H}^{2} \mathrm{~d} t+\varepsilon \gamma_{2} \int_{S}^{T}\left\|u_{2}^{\prime}\right\|_{H} \mathrm{~d} t+C_{\varepsilon} E\left(U^{\prime \prime}(S)\right) .
\end{aligned}
$$

Then, recalling that for all $v \in V_{j},\|v\|_{H}^{2} \leq 1 / \lambda_{j}\|v\|_{V_{j}}^{2}, j=1,2$, and adding (3.26) and (3.28), we obtain, for all $\varepsilon>0$,

$$
\begin{aligned}
\left(\frac{1}{2}-\gamma_{2} p^{+}\right) \int_{S}^{T}\left\|u_{1}^{\prime}\right\|_{H}^{2} \mathrm{~d} t & +\left(\frac{1}{2}-\frac{\beta_{2} \delta\left(p^{+}\right)^{2} p^{-}}{\lambda_{1}}\right) \int_{S}^{T}\left\|u_{1}\right\|_{V_{1}}^{2} \mathrm{~d} t+\left(\frac{\delta p^{-}}{2}-\varepsilon \gamma_{2}\right) \int_{S}^{T}\left\|u_{2}^{\prime}\right\|_{H}^{2} \mathrm{~d} t \\
& +\left(\frac{\delta p^{-}}{2}-\frac{\beta_{1} \delta^{2}\left(p^{+}\right)^{2}}{\lambda_{2}}\right) \int_{S}^{T}\left\|u_{2}\right\|_{V_{2}}^{2} \mathrm{~d} t \leq C E(U(S))+C E\left(U^{\prime}(S)\right)+C_{\varepsilon} E\left(U^{\prime \prime}(S)\right) .
\end{aligned}
$$

We now set $p_{*}=\min \left\{\frac{1}{2 \gamma_{2}}, \lambda_{2}\right\}>0$ and $\delta_{*}=\delta_{*}\left(p^{+}, p^{-}\right)=\min \left\{\frac{\lambda_{1}}{2 \beta_{2}\left(p^{+}\right)^{2} p^{-}}, \frac{\lambda_{2} p^{-}}{2 \beta_{1}\left(p^{+}\right)^{2}}, \frac{\lambda_{1}}{p^{+}}\right\}>0$. Then for all $p^{+} \in\left(0, p_{*}\right)$ and $\delta \in\left(0, \delta_{*}\right)$, one can choose $0<\varepsilon<\frac{\delta p^{-}}{2 \gamma_{2}}$, so that the following bound on the energy holds for all $0 \leq S \leq T$ and $U^{0} \in \mathcal{D}\left(\mathcal{A}_{P, \delta}^{2}\right)$,

$$
\begin{aligned}
\int_{S}^{T} E(U(t)) \mathrm{d} t & \leq C\left(\delta, p^{+}\right) \int_{S}^{T}\left(\left\|u_{1}^{\prime}(t)\right\|_{H}^{2}+\left\|u_{1}(t)\right\|_{V}^{2} \mathrm{~d} t+\left\|u_{2}^{\prime}(t)\right\|_{H}^{2}+\left\|u_{2}(t)\right\|_{V}^{2}\right) \mathrm{d} t \\
& \leq C\left(\delta, p^{+}, p^{-}\right)\left(E(U(S))+E\left(U^{\prime}(S)\right)+E\left(U^{\prime \prime}(S)\right)\right)
\end{aligned}
$$


from (3.29) and the choice of $p_{*}$ and $\delta_{*}$. Using now Lemma 3.1, we obtain

$$
E(U(t)) \leq \frac{C_{n}}{t^{n}} \sum_{i=0}^{2 n} E\left(U^{(i)}(0)\right) \quad \forall t>0, \quad \forall U^{0} \in \mathcal{D}\left(\mathcal{A}_{P, \delta}^{2 n}\right),
$$

and Theorem 2.7 is proved.

\section{Applications}

We now apply the results of Theorems 2.4 and 2.7 to different second order coupled systems. In each of the following sections, we first explain how the problem can be formulated in the abstract setting of Section 2.1. All these systems are well-posed in the spaces we choose, according to Proposition 2.2. Hence, we only have to check that assumptions $(A 1)-(A 4)$ hold in order to apply Theorem 2.4 or Theorem 2.7 and obtain the expected stability results. This strategy shall be followed in Section 4.1 to address internal stabilization of coupled wave equations, in Section 4.2 to address boundary stabilization of coupled wave equations, and in Section 4.3 to address internal stabilization of coupled plate equations. For the sake of brevity, we do not treat the case of boundary stabilization of coupled plate equations. However, one can prove as well that Theorem 2.7 can be applied in this case.

\subsection{Internal stabilization of locally coupled wave equations}

Here, we prove Theorem 1.2 in the context presented in the introduction. We recall that $\Omega \subset \mathbb{R}^{N}, \Gamma=\partial \Omega$ is of class $\mathcal{C}^{2}$, and consider the evolution problem (1.3). We take $H=L^{2}(\Omega), V=H_{0}^{1}(\Omega)$ with the usual inner products and norms. We moreover take for $B$ and $P$ respectively the multiplication in $L^{2}$ by the functions $b, p \in L^{\infty}(\Omega)$, satisfying

$$
\left\{\begin{array}{l}
0 \leq b \leq b^{+} \text {and } 0 \leq p \leq p^{+} \text {on } \Omega \\
b \geq b^{-}>0 \text { on } \omega_{b} \\
p \geq p^{-}>0 \text { on } \omega_{p}
\end{array}\right.
$$

for $\omega_{b}$ and $\omega_{p}$ two open subsets of $\Omega$, satisfying the PMGC. As a consequence, assumption $(A 4 b)$ is satisfied and assumption $(A 1)$ is fulfilled taking for $\Pi_{P}$ the multiplication by $\mathbb{1}_{\omega_{p}}$. It only remains to check assumptions $(A 2)$ and $(A 3)$, that are consequences of the following lemma.

Lemma 4.1. Let $\omega$ be a subset of $\Omega$ satisfying the PMGC. Then, there exist $\alpha, \beta, \gamma>0$ such that for all $f \in \mathcal{C}^{1}\left(\mathbb{R}^{+} ; L^{2}(\Omega)\right)$ and all $0 \leq S \leq T$, the solution $u$ of

$$
\begin{cases}u^{\prime \prime}-\Delta u=f & \text { in }(0,+\infty) \times \Omega, \\ u=0 & \text { on }(0,+\infty) \times \Gamma, \\ \left(u, u^{\prime}\right)(0, \cdot)=\left(u^{0}(\cdot), u^{1}(\cdot)\right) & \in H_{0}^{1}(\Omega) \times L^{2}(\Omega),\end{cases}
$$

satisfies, with $e(t)=1 / 2\left(\left\|u^{\prime}\right\|_{L^{2}(\Omega)}^{2}+\|\nabla u\|_{L^{2}(\Omega)}^{2}\right)$, the inequality

$$
\int_{S}^{T} e(t) \mathrm{d} t \leq \alpha(e(S)+e(T))+\beta \int_{S}^{T}\|f(t)\|_{L^{2}(\Omega)}^{2} \mathrm{~d} t+\gamma \int_{S}^{T}\left\|\mathbb{1}_{\omega} u^{\prime}(t)\right\|_{L^{2}(\Omega)}^{2} \mathrm{~d} t .
$$

This lemma direcly yields $(A 2)$, since $\omega_{p}$ is supposed to satisfy the PMGC. To prove $(A 3)$, we note that the solution $u$ of $(4.1)$ is $\mathcal{C}^{1}\left(\mathbb{R}^{+} ; L^{2}(\Omega)\right)$ and apply the lemma to $u=u_{1}, \omega=\omega_{b}$ and $f=f_{1}-b u_{1}^{\prime} \in \mathcal{C}^{1}\left(\mathbb{R}^{+} ; L^{2}(\Omega)\right)$. 
This yields

$$
\begin{aligned}
\int_{S}^{T} e_{1}(t) \mathrm{d} t & \leq \alpha\left(e_{1}(S)+e_{1}(T)\right)+\beta \int_{S}^{T}\left\|f_{1}(t)-b u_{1}^{\prime}(t)\right\|_{L^{2}(\Omega)}^{2} \mathrm{~d} t+\gamma \int_{S}^{T}\left\|\mathbb{1}_{\omega_{b}} u_{1}^{\prime}(t)\right\|_{L^{2}(\Omega)}^{2} \mathrm{~d} t \\
& \leq \alpha\left(e_{1}(S)+e_{1}(T)\right)+2 \beta \int_{S}^{T}\left\|f_{1}(t)\right\|_{L^{2}(\Omega)}^{2}+\left(2 \beta+\frac{\gamma}{\left(b^{-}\right)^{2}}\right) \int_{S}^{T}\left\|b u_{1}^{\prime}(t)\right\|_{L^{2}(\Omega)}^{2} \mathrm{~d} t
\end{aligned}
$$

which is $(A 3)$ with $\beta_{1}=2 \beta$ and $\gamma_{1}=\left(2 \beta+\frac{\gamma}{\left(b^{-}\right)^{2}}\right)$.

Now applying Theorem 2.4, we have proved the following theorem.

\section{Theorem 4.2.}

(i) Suppose that $\omega_{b}$ and $\omega_{p}$ satisfy the PMGC. Then there exists $p_{*} \in(0, \lambda]$ such that for all $0<p^{+}<p_{*}$ there exists $\delta_{*}=\delta_{*}\left(p^{+}, p^{-}\right) \in\left(0, \frac{\lambda}{p^{+}}\right]$, such that for all $\delta \in\left(0, \delta_{*}\right)$, the solution $U=\left(u_{1}, u_{2}, u_{1}^{\prime}, u_{2}^{\prime}\right)$ of (1.3) satisfies for $n \in \mathbb{N}$, for some $C_{n}>0$,

$$
E(U(t)) \leq \frac{C_{n}}{t^{n}} \sum_{i=0}^{n} E\left(U^{(i)}(0)\right) \quad \forall t>0, U^{0}=\left(u_{1}^{0}, u_{2}^{0}, u_{1}^{1}, u_{2}^{1}\right) \in \mathcal{D}\left(\mathcal{A}_{P, \delta}^{n}\right)
$$

where $\mathcal{D}\left(\mathcal{A}_{P, \delta}^{n}\right)$ is defined in (2.4). Besides, if $U^{0} \in \mathcal{H}=\left(H_{0}^{1}\right)^{2} \times\left(L^{2}\right)^{2}$, then $E(U(t))$ converges to zero as $t$ goes to infinity.

(ii) If moreover either $\omega_{b} \subset \omega_{p}$ or $\omega_{p} \subset \omega_{b}$, then the result holds for $\delta_{*}=\frac{\lambda}{p^{+}}$.

Theorem 1.2 is now a corollary of Theorem 4.2 in the case of smooth coefficients since Lemma 2.6 allows us to explicit the spaces $\mathcal{D}\left(\mathcal{A}_{P, \delta}^{n}\right)$.

Proof of Lemma 4.1. We here prove the energy estimate (4.2) for the solutions of (4.1), using the piecewise multiplier method. We proceed as in $[6,28]$. The subset $\omega$ satisfies the PMGC. Hence, denoting by $\Omega_{j}$ and $x_{j}, j=1 \ldots J$ the sets and the points given by the PMGC, we have $\omega \supset \mathcal{N}_{\varepsilon}\left(\bigcup_{j} \gamma_{j}\left(x_{j}\right) \cup\left(\Omega \backslash \bigcup_{j} \Omega_{j}\right)\right) \cap \Omega$. In this expression, $\mathcal{N}_{\varepsilon}(\mathcal{O})=\left\{x \in \mathbb{R}^{N}, d(x, \mathcal{O}) \leq \varepsilon\right\}$ with $d(\cdot, \mathcal{O})$ the usual euclidian distance to the subset $\mathcal{O}$ of $\mathbb{R}^{N}$, and $\gamma_{j}\left(x_{j}\right)=\left\{x \in \Gamma_{j},\left(x-x_{j}\right) \cdot \nu_{j}(x)>0\right\}$, where $\nu_{j}$ denotes the outward unit normal to $\Gamma_{j}=\partial \Omega_{j}$. Let $\varepsilon_{0}<\varepsilon_{1}<\varepsilon_{2}<\varepsilon$ and define

$$
Q_{i}=\mathcal{N}_{\varepsilon_{i}}\left(\bigcup_{j} \gamma_{j}\left(x_{j}\right) \cup\left(\Omega \backslash \bigcup_{j} \Omega_{j}\right)\right), \quad i=0,1,2 .
$$

Since $\left(\overline{\Omega_{j}} \backslash Q_{1}\right) \cap \overline{Q_{0}}=\emptyset$, we can construct a function $\psi_{j} \in \mathcal{C}_{0}^{\infty}\left(\mathbb{R}^{N}\right)$ which satisfies

$$
0 \leq \psi_{j} \leq 1, \quad \psi_{j}=1 \text { on } \overline{\Omega_{j}} \backslash Q_{1}, \quad \psi_{j}=0 \text { on } Q_{0} .
$$

For $m_{j}(x)=x-x_{j}$, we define the $\mathcal{C}^{1}$ vector field on $\Omega$ :

$$
h(x)= \begin{cases}\psi_{j}(x) m_{j}(x) & \text { if } x \in \Omega_{j} \\ 0 & \text { if } x \in \Omega \backslash \cup_{j} \Omega_{j} .\end{cases}
$$

Multiplying (4.1) by the multiplier $h \cdot \nabla u$ and integrating on $(S, T) \times \Omega_{j}$, we obtain

$$
\int_{S}^{T} \int_{\Omega_{j}} h(x) \cdot \nabla u\left(u^{\prime \prime}-\Delta u-f\right) \mathrm{d} x \mathrm{~d} t=0 .
$$


For the sake of concision, we will omit the $\mathrm{d} x \mathrm{~d} t$ in the following integrals. This gives, after integration by parts

$$
\begin{aligned}
\int_{S}^{T} \int_{\Gamma_{j}}\left(\partial_{\nu_{j}} u h \cdot \nabla u+\frac{1}{2}(h \cdot \nu)\left(u^{\prime 2}-|\nabla u|^{2}\right)\right) & {\left[\int_{\Omega_{j}} u^{\prime} h \cdot \nabla u\right]_{S}^{T} } \\
& +\int_{S}^{T} \int_{\Omega_{j}}\left(\frac{1}{2} \operatorname{div} h\left(u^{\prime 2}-|\nabla u|^{2}\right)+\sum_{i, k} \frac{\partial h_{k}}{\partial x_{i}} \frac{\partial u}{\partial x_{i}} \frac{\partial u}{\partial x_{k}}-f h \cdot \nabla u\right) .
\end{aligned}
$$

Thanks to the choice of $\psi_{j}$, only the boundary term on $\left(\Gamma_{j} \backslash \gamma_{j}\left(x_{j}\right)\right) \cap \Gamma$ is nonvanishing in the left hand side of (4.3). But on this part of the boundary $u=0$, so that $u^{\prime}=0$ and $\nabla u=\partial_{\nu} u \nu=\partial_{\nu_{j}} u \nu_{j}$. Hence, the left hand side of (4.3) reduces to

$$
\frac{1}{2} \int_{S}^{T} \int_{\left(\Gamma_{j} \backslash \gamma_{j}\left(x_{j}\right)\right) \cap \Gamma}\left(\partial_{\nu_{j}} u\right)^{2} \psi_{j}\left(m_{j} \cdot \nu_{j}\right) \leq 0 .
$$

Therefore, since $\psi_{j}=0$ on $Q_{0}$ and thanks to the above inequality used in (4.3), we deduce that

$$
\left[\int_{\Omega_{j}} u^{\prime} h \cdot \nabla u\right]_{S}^{T}+\int_{S}^{T} \int_{\Omega_{j} \backslash Q_{0}}\left(\frac{1}{2} \operatorname{div} h\left(u^{\prime 2}-|\nabla u|^{2}\right)+\sum_{i, k} \frac{\partial h_{k}}{\partial x_{i}} \frac{\partial u}{\partial x_{i}} \frac{\partial u}{\partial x_{k}}-f h \cdot \nabla u\right) \leq 0 .
$$

Using $\psi_{j}=1$ on $\overline{\Omega_{j}}-Q_{1}$ and summing the resulting inequalities on $j$, we obtain

$$
\begin{aligned}
{\left[\int_{\Omega} u^{\prime} h \cdot \nabla u\right]_{S}^{T}+\int_{S}^{T} } & \int_{\Omega \backslash Q_{1}} \frac{1}{2}\left(N u^{\prime 2}+(2-N)|\nabla u|^{2}\right)-\int_{S}^{T} \int_{\Omega} f h \cdot \nabla u \\
\leq-\sum_{j} \int_{S}^{T}\left[\int_{\Omega_{j} \cap Q_{1}} \frac{1}{2} \operatorname{div}\left(\psi_{j} m_{j}\right)\left(u^{\prime 2}-|\nabla u|^{2}\right)+\sum_{i, k} \frac{\partial h_{k}}{\partial x_{i}} \frac{\partial u}{\partial x_{i}} \frac{\partial u}{\partial x_{k}}\right] & \leq C \int_{S}^{T} \int_{\Omega_{1} Q_{1}}\left(u^{\prime 2}+|\nabla u|^{2}\right),
\end{aligned}
$$

where $C$ is a positive constant which depends only on $\psi_{j}$ and $m_{j}$. We now use the second multiplier $u(N-1) / 2$ and therefore evaluate the term

$$
\frac{N-1}{2} \int_{S}^{T} \int_{\Omega} u\left(u^{\prime \prime}-\Delta u-f\right)=0
$$

Hence, one has

$$
\left.\frac{N-1}{2}\left[\int_{\Omega} u u^{\prime}\right]_{S}^{T}+\frac{N-1}{2} \int_{S}^{T} \int_{\Omega}|\nabla u|^{2}-u^{\prime 2}-u f\right)=0 .
$$

We set $M(u)=h \cdot \nabla u+\frac{N-1}{2} u$. Adding (4.5) to (4.4), we obtain

$$
\int_{S}^{T} e(t) \mathrm{d} t \leq C \int_{S}^{T} \int_{\Omega \cap Q_{1}}|\nabla u|^{2}+C \int_{S}^{T} \int_{\Omega \cap Q_{1}} u^{\prime 2}-\left[\int_{\Omega} M(u) u^{\prime}\right]_{S}^{T}+\int_{S}^{T} \int_{\Omega} M(u) f .
$$

We now estimate the terms on the right hand side of (4.6) as follows. First, we have

$$
\left|\left[\int_{\Omega} M(u) u^{\prime}\right]_{S}^{T}\right| \leq C(e(S)+e(T)) .
$$


Second, we estimate the last term of (4.6) as follows

$$
\left|\int_{S}^{T} \int_{\Omega} M(u) f\right| \leq \frac{C}{\mu} \int_{S}^{T} \int_{\Omega}|f|^{2}+\mu \int_{S}^{T} e(t) \mathrm{d} t \quad \forall \mu>0 .
$$

The difficulty is now to estimate the first term on the right hand side of (4.6). We just follow techniques developed in [28]. We give the steps for the sake of completeness. First, we dominate the integral of $|\nabla u|^{2}$ in (4.6) by the integral of $u^{2}$ and $u^{\prime 2}$, localized inside the region of observation. Second, in (4.10) below, we bound the integral of $u^{2}$ in terms of the integral of $u^{\prime 2}$ in the same region. Since $\overline{\mathbb{R}^{N} \backslash Q_{2}} \cap \overline{Q_{1}}=\emptyset$, there exists a function $\xi \in \mathcal{C}_{0}^{\infty}\left(\mathbb{R}^{N}\right)$ such that

$$
0 \leq \xi \leq 1, \quad \xi=1 \text { on } Q_{1}, \quad \xi=0 \text { on } \mathbb{R}^{N} \backslash Q_{2} .
$$

Multiplying (4.1) by $\xi u$ and integrating on $[S, T] \times \Omega$, we obtain after integrations by parts

$$
\int_{S}^{T} \int_{\Omega} \xi|\nabla u|^{2}=\int_{S}^{T} \int_{\Omega}\left(\xi\left|u^{\prime}\right|^{2}+\frac{1}{2} \Delta \xi u^{2}\right)-\left[\int_{\Omega} \xi u u^{\prime}\right]_{S}^{T}+\int_{S}^{T} \int_{\Omega} \xi u f .
$$

We thus have

$$
\int_{S}^{T} \int_{\Omega \cap Q_{1}}|\nabla u|^{2} \leq C \int_{S}^{T} \int_{\Omega \cap Q_{2}}\left(\left|u^{\prime}\right|^{2}+u^{2}+|f|^{2}\right)+C(e(S)+e(T)) .
$$

Since $\overline{\mathbb{R}^{N} \backslash \omega} \cap \overline{Q_{2}}=\emptyset$, there exists a function $\beta \in \mathcal{C}_{0}^{\infty}\left(\mathbb{R}^{N}\right)$ such that

$$
0 \leq \beta \leq 1, \quad \beta=1 \text { on } Q_{2}, \quad \beta=0 \text { on } \mathbb{R}^{N} \backslash \omega .
$$

Proceeding as in [15], we fix $t$ and consider the solution $z$ of the following elliptic problem

$$
\begin{cases}\Delta z=\beta(x) u & \text { in } \Omega \\ z=0 & \text { on } \Gamma .\end{cases}
$$

Hence, $z$ and $z^{\prime}$ satisfy the following estimates

$$
\|z\|_{L^{2}(\Omega)} \leq C\|u\|_{L^{2}(\Omega)}, \quad \text { and } \quad\left\|z^{\prime}\right\|_{L^{2}(\Omega)}^{2} \leq C \int_{\Omega} \beta\left|u^{\prime}\right|^{2} .
$$

Multiplying (4.1) by $z$ and integrating on $[S, T] \times \Omega$, we obtain after integrations by parts

$$
\int_{S}^{T} \int_{\Omega} \beta u^{2}=\left[\int_{\Omega} z u^{\prime}\right]_{S}^{T}+\int_{S}^{T} \int_{\Omega}-z^{\prime} u^{\prime}-z f .
$$

Hence, using the estimates (4.9) in the above relation, we obtain for all $\eta>0$

$$
\int_{S}^{T} \int_{\Omega \cap Q_{2}}|u|^{2} \leq \frac{C}{\eta} \int_{S}^{T} \int_{\omega}\left|u^{\prime}\right|^{2}+\frac{C}{\eta} \int_{S}^{T} \int_{\Omega}|f|^{2}+\eta \int_{S}^{T} e+C(e(S)+e(T)) .
$$

Combined with the estimates (4.7), (4.8) and (4.10) in (4.6), this gives for all $\mu>0$

$$
\int_{S}^{T} e \leq C(e(S)+e(T))+C \mu \int_{S}^{T} e+\frac{C}{\mu} \int_{S}^{T}\left[\int_{\omega}\left|u^{\prime}\right|^{2}+\int_{\Omega}|f|^{2}\right] .
$$


Choosing $\mu$ sufficiently small, we finally have

$$
\int_{S}^{T} e \leq C(e(S)+e(T))+C \int_{S}^{T}\left[\int_{\omega}\left|u^{\prime}\right|^{2}+\int_{\Omega}|f|^{2}\right]
$$

and the lemma is proved.

\subsection{Boundary stabilization of locally coupled wave equations}

Here, we are interested in boundary stabilization. The results given generalize the ones of $[2,3]$ where the case of constant coupling has been considered. Given $\Omega \subset \mathbb{R}^{N}$ and $\Gamma=\partial \Omega$ of class $\mathcal{C}^{2}$ we shall use the following boundary multiplier geometric condition (BMGC).

Definition 4.3 (BMGC). Let $\left\{\Sigma_{1}, \Sigma_{0}\right\}$ be a partition of $\Gamma$ such that $\bar{\Sigma}_{1} \cap \bar{\Sigma}_{0}=\emptyset$. We say that $\left\{\Sigma_{1}, \Sigma_{0}\right\}$ satisfies the BMGC if there exists $x_{0} \in \mathbb{R}^{N}$ such that $m \cdot \nu \leq 0$ on $\Sigma_{0}$ and $m \cdot \nu \geq m^{-}>0$ on $\Sigma_{1}$, where $m(x)=x-x_{0}$.

The most simple situation covered by this condition is the case where $\Omega$ is star-shaped with respect to $x_{0}$. In this case $\Sigma_{0}=\emptyset$ and $\Sigma_{1}=\Gamma$. Another interesting and somehow more general situation is the case where $\Omega=\Omega_{1} \backslash \overline{\Omega_{2}}$, with $\Omega_{2}$ and $\Omega_{1}$ two open subset of $\mathbb{R}^{N}$, both star-shaped with respect to $x_{0}$, and such that $\overline{\Omega_{2}} \subset \Omega_{1}$. In this case, $\partial \Omega=\partial \Omega_{1} \cup \partial \Omega_{2}$ with a disjoint union, $\Sigma_{0}=\partial \Omega_{2}$ and $\Sigma_{1}=\partial \Omega_{1}$ satisfy the BMGC.

For $\Gamma_{b} \subset \Gamma$, and $\Gamma_{0}=\Gamma \backslash \Gamma_{b}$, we consider the following coupled stabilization problem

$$
\begin{cases}u_{1}^{\prime \prime}-\Delta u_{1}+\delta p u_{2}=0 & \text { in }(0,+\infty) \times \Omega, \\ u_{2}^{\prime \prime}-\Delta u_{2}+p u_{1}=0 & \text { in }(0,+\infty) \times \Omega, \\ \frac{\partial u_{1}}{\partial \nu}+m \cdot \nu\left(l u_{1}+b u_{1}^{\prime}\right)=0 & \text { on }(0,+\infty) \times \Gamma_{b}, \\ u_{1}=0 & \text { on }(0,+\infty) \times \Gamma_{0}, \\ u_{2}=0 & \text { on }(0,+\infty) \times \Gamma, \\ u_{j}(0, \cdot)=u_{j}^{0}(\cdot), u_{j}^{\prime}(0, \cdot)=u_{j}^{1}(\cdot), j=1,2 & \text { in } \Omega,\end{cases}
$$

where $l$ is a non-negative function on $\Gamma_{b}$. Note that we added $m \cdot \nu$, where $m(x)=x-x_{0}$ in the stabilization term to avoid some technical estimates. This term can be removed, provided that we do some more assumptions on the functions $b$ and $l$. Here we make the following assumptions on the coefficients $b$ and $p$

$$
\left\{\begin{array}{l}
0 \leq b \leq b^{+} \text {on } \Gamma, \text { and } b \geq b^{-}>0 \text { on } \Gamma_{b} \\
0 \leq p \leq p^{+} \text {on } \Omega, \text { and } p \geq p^{-}>0 \text { on } \omega_{p}
\end{array}\right.
$$

Moreover we set $H_{\Gamma_{0}}^{1}(\Omega)=\left\{u \in H^{1}(\Omega), u=0\right.$ on $\left.\Gamma_{0}\right\}$, and we shall assume for the sake of clarity that $l \neq 0$ or meas $\left(\Gamma_{0}\right) \neq 0$. We take $H=L^{2}(\Omega)$ and $V_{1}=H_{\Gamma_{0}}^{1}(\Omega)$ equipped respectively with the $L^{2}$ inner product and the inner product $(u, z)_{V_{1}}=\int_{\Omega} \nabla u \cdot \nabla z+\int_{\Gamma_{b}}(m \cdot \nu l u z)$ and the corresponding norms. Moreover we take $V_{2}=H_{0}^{1}(\Omega)$ equipped with the inner product $(u, z)_{V_{2}}=\int_{\Omega} \nabla u \cdot \nabla z$ and the associated norm. We define the duality mappings $A_{1}$ and $A_{2}$ as in Section 2.1. We also define the continuous linear operator $B$ from $V_{1}$ to $V_{1}^{\prime}$ by

$$
\langle B u, z\rangle_{V_{1}^{\prime}, V_{1}}=\int_{\Gamma_{b}} m \cdot \nu b u z \mathrm{~d} \gamma
$$

that satisfies (2.2). As in Section 4.2, we take for $P$ the multiplication in $L^{2}$ by the function $p \in L^{\infty}$. With these notations, system (4.11) can be rewritten under the form (1.5).

Theorem 4.4. Suppose that $\omega_{p}$ satisfies the PMGC and $\left\{\Gamma_{b}, \Gamma_{0}\right\}$ satisfies the BMGC. Then there exists $p_{*} \in$ $(0, \lambda]$ such that for all $0<p^{+}<p_{*}$ there exists $\delta_{*}=\delta_{*}\left(p^{+}, p^{-}\right) \in\left(0, \frac{\lambda}{p^{+}}\right]$, such that for all $\delta \in\left(0, \delta_{*}\right)$, the 
solution $U=\left(u_{1}, u_{2}, u_{1}^{\prime}, u_{2}^{\prime}\right)$ of (4.11) satisfies, for $n \geq 1$, for some $C_{n}>0$,

$$
E(U(t)) \leq \frac{C_{n}}{t^{n}} \sum_{i=0}^{2 n} E\left(U^{(i)}(0)\right) \quad \forall t>0, \quad \forall U^{0}=\left(u_{1}^{0}, u_{2}^{0}, u_{1}^{1}, u_{2}^{1}\right) \in \mathcal{D}\left(\mathcal{A}_{P, \delta}^{2 n}\right) .
$$

Besides, if $U^{0} \in \mathcal{H}=H_{\Gamma_{0}}^{1} \times H_{0}^{1} \times\left(L^{2}\right)^{2}$, then $E(U(t))$ converges to zero as $t$ goes to infinity.

We recall that the operator $\mathcal{A}_{P, \delta}$ is defined in (2.4). As opposed to the results for internal damping, we do not have here a simple expression of $\mathcal{D}\left(\mathcal{A}_{P, \delta}^{2 n}\right)$ in terms of Sobolev spaces.

To prove this theorem, we just need to check that the assumptions $(A 1)-(A 4 u)$ are satisfied and then apply Theorem 2.7 in a convenient setting. First, assumption $(A 1)$ is satisfied with $\Pi_{P}$ the multiplication by $\mathbb{1}_{\omega_{p}}$, and assumption $(A 2)$ is a consequence of Lemma 4.5 as in Section 4.1, since the internal coupling is here the same.

We now check assumption $(A 4 u)$ and follow the lines of [3]. For the sake of clarity, we identify $i(\phi)$ with $\phi$ for $\phi \in V_{2}$ (where $i$ is the canonical injection from $V_{2}$ in $V_{1}$ ). We first remark that the first equality in assumption $(A 4 u)$ is satisfied thanks to the definition of $B$ and $V_{2}$. We define $\Pi_{V}$ and $A_{2}$ as in Section 2.1. Then, $\Pi_{V} u_{1}$ is the weak solution of

$$
\left\{\begin{array}{l}
-\Delta \Pi_{V} u_{1}=-\Delta u_{1} \quad \text { in } \Omega \\
\Pi_{V} u_{1} \in V_{2}
\end{array}\right.
$$

and $A_{2}$ is defined by

$$
\left\langle A_{2} \phi, \psi\right\rangle_{V_{2}^{\prime}, V_{2}}=\int_{\Omega} \nabla \phi \cdot \nabla \psi \mathrm{d} x, \quad \forall \psi, \phi \in V_{2} .
$$

We now check the second relation in $(A 4 u)$. For this, we set $z=u_{1}-\Pi_{V} u_{1}$, so that $z$ is the weak solution of

$$
\begin{cases}-\Delta z=0 & \text { in } \Omega \\ z=u_{1}, & \text { on } \Gamma\end{cases}
$$

By elliptic regularity, we deduce that there exists a constant $c>0$ such that $\|z\|_{H} \leq c\left\|u_{1 \mid \Gamma_{b}}\right\|_{L^{2}\left(\Gamma_{b}\right)}$. Since we assume the BMGC, $m \cdot \nu b \geq m^{-} b^{-}>0$ on $\Gamma_{b}$, there exists then $\beta>0$ such that

$$
\|z\|_{H}^{2} \leq \beta\left\langle B u_{1}, u_{1}\right\rangle_{V_{1}^{\prime}, V_{1}} \quad \forall u_{1} \in V_{1},
$$

and $(A 4 u)$ is satisfied.

The last assumption $(A 3)$ is a direct consequence of the following lemma. Theorem 4.4 follows then from Theorem 2.7.

Lemma 4.5. Suppose that $\left\{\Gamma_{b}, \Gamma_{0}\right\}$ satisfies the BMGC. Then, there exist $\alpha, \beta, \gamma>0$ such that for all $f \in$ $\mathcal{C}^{1}\left(\mathbb{R}^{+} ; L^{2}(\Omega)\right)$ and all $0 \leq S \leq T$, the solution $u$ of

$$
\begin{cases}u^{\prime \prime}-\Delta u=f & \text { in }(0,+\infty) \times \Omega, \\ \frac{\partial u}{\partial \nu}+m \cdot \nu\left(l u+b u^{\prime}\right)=0 & \text { on }(0,+\infty) \times \Gamma_{b}, \\ u=0 & \text { on }(0,+\infty) \times \Gamma_{0}, \\ \left(u, u^{\prime}\right)(0, \cdot)=\left(u^{0}(\cdot), u^{1}(\cdot)\right) & \in H_{0}^{1}(\Omega) \times L^{2}(\Omega),\end{cases}
$$

satisfies, with $e(t)=1 / 2\left(\left\|u^{\prime}\right\|_{L^{2}(\Omega)}^{2}+\|\nabla u\|_{L^{2}(\Omega)}^{2}+\int_{\Gamma_{b}} m \cdot \nu l u^{2} \mathrm{~d} \gamma\right)$, the inequality

$$
\int_{S}^{T} e(t) \mathrm{d} t \leq \alpha(e(S)+e(T))+\beta \int_{S}^{T}\|f(t)\|_{L^{2}(\Omega)}^{2} \mathrm{~d} t+\gamma \int_{S}^{T} \int_{\Gamma_{b}} m \cdot \nu b u^{\prime 2} \mathrm{~d} x \mathrm{~d} t .
$$


Proof. Proceeding as in [20], Theorem 8.6, we first use the multiplier $M u=m(x) \cdot \nabla u+\frac{(N-1)}{2} u$ and set $R=\sup \{|m(x)|, x \in \Omega\}$. Multiplying (4.12) by $M u$, we obtain after integrations by parts

$$
\begin{aligned}
\int_{S}^{T} e(t) \mathrm{d} t=\int_{S}^{T} \int_{\Omega} f M u-\left[\int_{\Omega} u^{\prime}\right. & M u]_{S}^{T}+\frac{1}{2} \int_{S}^{T} \int_{\Gamma_{0}} m \cdot \nu\left|\frac{\partial u}{\partial \nu}\right|^{2} \mathrm{~d} \gamma \mathrm{d} t \\
& \quad \int_{S}^{T} \int_{\Gamma_{b}} m \cdot \nu\left(\frac{1}{2} u^{\prime 2}-\frac{1}{2}|\nabla u|^{2}-\left(l u+b u^{\prime}\right)\left(m \cdot \nabla u+\frac{N-1}{2} u\right)+l \frac{u^{2}}{2}\right) .
\end{aligned}
$$

Setting $h=\frac{u^{\prime 2}-|\nabla u|^{2}}{2}+R\left|l u+b u^{\prime}\right||\nabla u|+|2-N| l \frac{u^{2}}{2}-b u u^{\prime} \frac{(N-1)}{2}$, this yields, for all $\eta>0$,

$$
\int_{S}^{T} e(t) \mathrm{d} t \leq C(e(S)+e(T))+\frac{C}{\eta} \int_{S}^{T} \int_{\Omega} f^{2}+\eta \int_{S}^{T} e(t) \mathrm{d} t+\int_{S}^{T} \int_{\Gamma_{b}} m \cdot \nu h .
$$

We now estimate the term $h$. Since $b \geq b^{-}>0$ on $\Gamma_{b}$, we have for all $\varepsilon>0$,

$$
h \leq C\left(1+\frac{1}{\varepsilon}\right) b u^{\prime 2}+C l u^{2}+\varepsilon u^{2} .
$$

Concerning the last term, in this equation, we have the trace inequality

$$
\varepsilon \int_{S}^{T} \int_{\Gamma_{b}} m \cdot \nu u^{2} \leq C \varepsilon \int_{S}^{T}\left(\int_{\Omega} \frac{|\nabla u|^{2}}{2}+\int_{\Gamma_{b}} m \cdot \nu l u^{2}\right)
$$

Hence, for $\varepsilon$ and $\eta$ sufficiently small, using (4.16) and (4.15) in (4.14), we obtain

$$
\int_{S}^{T} e(t) \mathrm{d} t \leq C(e(T)+e(S))+C \int_{S}^{T} \int_{\Omega} f^{2}+C \int_{S}^{T} \int_{\Gamma_{b}} m \cdot \nu b u^{\prime 2}+C \int_{S}^{T} \int_{\Gamma_{b}} m \cdot \nu l u^{2} .
$$

It only remains to treat the last term in this inequality. For this, we use the method introduced in [15]. Let $z$ be the solution of the following elliptic problem:

$$
\begin{cases}\Delta z=0 & \text { in } \Omega \\ z=u & \text { on } \Gamma_{b} \\ z=0 & \text { on } \Gamma_{0}\end{cases}
$$

Note that this definition yields

$$
\int_{\Omega}|\nabla z|^{2}+z^{2} \leq C \int_{\Gamma_{b}} u^{2} \quad \text { and } \quad \int_{\Omega} \nabla z \cdot \nabla u=\int_{\Omega}|\nabla z|^{2} .
$$

We multiply (4.12) by $z$ and integrate on $(S, T) \times \Omega$. Integrating by parts and using (4.18) and the boundary conditions for $u$, we obtain

$$
\begin{aligned}
\int_{S}^{T} \int_{\Gamma_{b}} m \cdot \nu l u^{2}= & \int_{S}^{T} \int_{\Omega}\left(u^{\prime} z^{\prime}-|\nabla z|^{2}+f z\right)-\left[\int_{\Omega} u^{\prime} z\right]_{S}^{T}-\int_{S}^{T} m \cdot b u^{\prime} u \\
\leq & \varepsilon \int_{S}^{T} \int_{\Omega} u^{\prime 2}+\frac{C}{\varepsilon} \int_{S}^{T} \int_{\Omega} z^{\prime 2}+\frac{C}{\varepsilon} \int_{S}^{T} \int_{\Omega} f^{2}+C \varepsilon \int_{S}^{T} \int_{\Omega} z^{2} \\
& +C(e(T)+e(S))+\frac{C}{\varepsilon} \int_{S}^{T} \int_{\Gamma_{b}} m \cdot \nu b u^{\prime 2}+\varepsilon \int_{S}^{T} \int_{\Gamma_{b}} u^{2}
\end{aligned}
$$


for all $\varepsilon>0$. Now noting that

$$
\int_{S}^{T} \int_{\Omega} z^{\prime 2} \leq \int_{S}^{T} \int_{\Gamma_{b}} m^{-} b^{-} z^{\prime 2} \leq \int_{S}^{T} \int_{\Gamma_{b}} m \cdot \nu b z^{\prime 2}
$$

and

the estimate (4.19) yields, for all $\varepsilon>0$,

$$
\int_{S}^{T} \int_{\Omega} u^{\prime 2}+\int_{S}^{T} \int_{\Omega} z^{2}+\int_{S}^{T} \int_{\Gamma_{b}} u^{2} \leq C \int_{S}^{T} e(t) \mathrm{d} t
$$

$$
\int_{S}^{T} \int_{\Gamma_{b}} m \cdot \nu l u^{2} \leq C \varepsilon \int_{S}^{T} e(t) \mathrm{d} t+\frac{C}{\varepsilon} \int_{S}^{T} \int_{\Omega} f^{2}+C(e(T)+e(S))+\frac{C}{\varepsilon} \int_{S}^{T} \int_{\Gamma_{b}} m \cdot \nu b u^{\prime 2} .
$$

Finally, replacing this in (4.17) and taking $\varepsilon$ sufficiently small, we obtain

$$
\int_{S}^{T} e(t) \mathrm{d} t \leq C(e(T)+e(S))+C \int_{S}^{T} \int_{\Omega} f^{2}+C \int_{S}^{T} \int_{\Gamma_{b}} m \cdot \nu b u^{\prime 2}
$$

and the lemma is proved.

\subsection{Internal stabilization of locally coupled plate equations}

In this last application, we are concerned with a system of two weakly coupled plate equations. This generalizes the case of constant coupling investigated in [1]. Here, we assume that the boundary $\Gamma=\partial \Omega$ is at least of class $\mathcal{C}^{4}$ and we consider the following system:

$$
\begin{cases}u_{1}^{\prime \prime}+\Delta^{2} u_{1}+\delta p u_{2}+b u_{1}^{\prime}=0 & \text { in }(0,+\infty) \times \Omega, \\ u_{2}^{\prime \prime}+\Delta^{2} u_{2}+p u_{1}=0 & \text { in }(0,+\infty) \times \Omega, \\ u_{1}=0 \text { and } u_{2}=0 & \text { on }(0,+\infty) \times \Gamma, \\ \frac{\partial u_{1}}{\partial \nu}=0 \text { and } \frac{\partial u_{2}}{\partial \nu}=0 & \text { on }(0,+\infty) \times \Gamma, \\ u_{j}(0, \cdot)=u_{j}^{0}(\cdot), u_{j}^{\prime}(0, \cdot)=u_{j}^{1}(\cdot), j=1,2 & \text { in } \Omega .\end{cases}
$$

We take $H=L^{2}(\Omega)$, and $V_{1}=V_{2}=H_{0}^{2}(\Omega)$ endowed with the inner product $(y, z)_{H_{0}^{2}(\Omega)}=\int_{\Omega} \Delta y \Delta z \mathrm{~d} x$. Hence, $A=\Delta^{2}$ with Neumann and Dirichlet boundary conditions, $\mathcal{D}\left(A^{\frac{n}{2}}\right) \subset H^{2 n}(\Omega)$ is the domain of $A^{\frac{n}{2}}$ and $\lambda$ denotes its lowest eigenvalue. We moreover take for $B$ and $P$ respectively the multiplication in $L^{2}$ by the functions $b, p \in L^{\infty}(\Omega)$ satisfying, as in Section 4.1,

$$
\left\{\begin{array}{l}
0 \leq b \leq b^{+} \text {and } 0 \leq p \leq p^{+} \text {on } \Omega \\
b \geq b^{-}>0 \text { on } \omega_{b} \\
p \geq p^{-}>0 \text { on } \omega_{p}
\end{array}\right.
$$

for $\omega_{b}$ and $\omega_{p}$ two open subsets of $\Omega$. As for coupled waves, we have the following stability result.

\section{Theorem 4.6.}

(i) Suppose that $\omega_{b}$ and $\omega_{p}$ satisfy the PMGC. Then there exists $p_{*} \in(0, \lambda]$ such that for all $0<p^{+}<p_{*}$ there exists $\delta_{*}=\delta_{*}\left(p^{+}, p^{-}\right) \in\left(0, \frac{\lambda}{p^{+}}\right]$, such that for all $\delta \in\left(0, \delta_{*}\right)$, the solution $U=\left(u_{1}, u_{2}, u_{1}^{\prime}, u_{2}^{\prime}\right)$ 
of (4.20) satisfies for $n \in \mathbb{N}$, for some $C_{n}>0$,

$$
E(U(t)) \leq \frac{C_{n}}{t^{n}} \sum_{i=0}^{n} E\left(U^{(i)}(0)\right) \quad \forall t>0, U^{0}=\left(u_{1}^{0}, u_{2}^{0}, u_{1}^{1}, u_{2}^{1}\right) \in \mathcal{D}\left(\mathcal{A}_{P, \delta}^{n}\right)
$$

Besides, if $U^{0} \in\left(H_{0}^{2}\right)^{2} \times\left(L^{2}\right)^{2}$, then $E(U(t))$ converges to zero as $t$ goes to infinity.

(ii) If moreover either $\omega_{b} \subset \omega_{p}$ or $\omega_{p} \subset \omega_{b}$, then the result holds for $\delta_{*}=\frac{\lambda}{p^{+}}$.

We recall that the operator $\mathcal{A}_{P, \delta}$ is defined in (2.4). Under some smoothness assumptions on the coefficients $p$ and $b$, we can explicit the space $\mathcal{D}\left(\mathcal{A}_{P, \delta}^{n}\right)$ in terms of iterated domains of the bilaplacian with Dirichlet and Neumann boundary conditions, thanks to Lemma 2.6. This gives the following corollary.

\section{Corollary 4.7.}

(i) Suppose that $\omega_{b}$ and $\omega_{p}$ satisfy the PMGC. Then there exists $p_{*} \in(0, \lambda]$ such that for all $0<p^{+}<p_{*}$ there exists $\delta_{*}=\delta_{*}\left(p^{+}, p^{-}\right) \in\left(0, \frac{\lambda}{p^{+}}\right]$, such that for all $\delta \in\left(0, \delta_{*}\right)$, for all $b, p \in W^{2 q, \infty}(\Omega)$ satisfying (4.21), the solution $U=\left(u_{1}, u_{2}, u_{1}^{\prime}, u_{2}^{\prime}\right)$ of (4.20) satisfies, for $n \in \mathbb{N}, n \leq q$, for some $C_{n}>0$,

$$
E(U(t)) \leq \frac{C_{n}}{t^{n}} \sum_{i=0}^{n} E\left(U^{(i)}(0)\right) \quad \forall t>0, U^{0}=\left(u_{1}^{0}, u_{2}^{0}, u_{1}^{1}, u_{2}^{1}\right) \in \mathcal{D}\left(A^{\frac{n+1}{2}}\right)^{2} \times \mathcal{D}\left(A^{\frac{n}{2}}\right)^{2}
$$

Besides, if $U^{0} \in\left(H_{0}^{2}\right)^{2} \times\left(L^{2}\right)^{2}$, then $E(U(t))$ converges to zero as $t$ goes to infinity.

(ii) If moreover either $\omega_{b} \subset \omega_{p}$ or $\omega_{p} \subset \omega_{b}$, then the result holds for $\delta_{*}=\frac{\lambda}{p^{+}}$.

To prove Theorem 4.6, we only have to check that assumptions $(A 1)-(A 4 b)$ hold and use Theorem 2.4. From (4.21), assumption $(A 4 b)$ is satisfied and assumption $(A 1)$ is fulfilled, taking for $\Pi_{P}$ the multiplication in $L^{2}$ by $\mathbb{1}_{\omega_{p}}$. It only remains to check assumptions $(A 2)$ and $(A 3)$, that are consequences of the following lemma.

Lemma 4.8. Let $\omega$ be a subset of $\Omega$ satisfying the PMGC. Then, there exist $\alpha, \beta, \gamma>0$ such that for all $f \in \mathcal{C}^{1}\left(\mathbb{R}^{+} ; L^{2}(\Omega)\right)$ and all $0 \leq S \leq T$, the solution $u$ of

$$
\begin{cases}u^{\prime \prime}+\Delta^{2} u=f & \text { in }(0,+\infty) \times \Omega \\ u=0 \text { and } \frac{\partial u}{\partial \nu}=0 & \text { on }(0,+\infty) \times \Gamma \\ \left(u, u^{\prime}\right)(0, \cdot)=\left(u^{0}(\cdot), u^{1}(\cdot)\right) & \in H_{0}^{2}(\Omega) \times L^{2}(\Omega)\end{cases}
$$

satisfies, with $e(t)=1 / 2\left(\left\|u^{\prime}\right\|_{L^{2}(\Omega)}^{2}+\|\Delta u\|_{L^{2}(\Omega)}^{2}\right)$, the inequality

$$
\int_{S}^{T} e(t) \mathrm{d} t \leq \alpha(e(S)+e(T))+\beta \int_{S}^{T}\|f(t)\|_{L^{2}(\Omega)}^{2} \mathrm{~d} t+\gamma \int_{S}^{T}\left\|\mathbb{1}_{\omega} u^{\prime}(t)\right\|_{L^{2}(\Omega)}^{2} \mathrm{~d} t
$$

This lemma direcly yields $(A 2)$, since $\omega_{p}$ is supposed to satisfy the PMGC. Proving $(A 3)$ is done exactly as in Section 4.1, taking $u=u_{1}, \omega=\omega_{b}$ and $f=f_{1}-b u_{1}^{\prime}$ in Lemma 4.8. Theorem 4.6 is then a consequence of Theorem 2.4.

Proof. We give here the details of the piecewise multiplier method for a plate equation, following [7], so that the proof is selfcontained. We denote by $\mathcal{N}_{\varepsilon}\left(\cup_{j} \gamma_{j}\left(x_{j}\right) \cup\left(\Omega \backslash \cup_{j} \Omega_{j}\right)\right)$ the neighborhood given by the PMGC (see Def. 1.1 in the Introduction). Let $0<\varepsilon_{0}<\varepsilon_{1}<\varepsilon_{2}<\varepsilon$ and define for $i=0,1,2$

$$
Q_{i}=\mathcal{N}_{\varepsilon_{i}}\left(\cup_{j} \gamma_{j}\left(x_{j}\right) \cup\left(\Omega \backslash \cup_{j} \Omega_{j}\right)\right)
$$


where $\Omega_{j}, x_{j}$ and $\gamma_{j}\left(x_{j}\right)$ are given by the PMGC. Recall that $\Gamma_{j}=\partial \Omega_{j}$ and $m_{j}(x)=x-x_{j}$. Since $\left(\overline{\Omega_{j}} \backslash Q_{1}\right) \cap \overline{Q_{0}}=$ $\emptyset$, we can construct a function $\psi_{j} \in \mathcal{C}_{0}^{\infty}\left(\mathbb{R}^{N}\right)$ which satisfies

$$
0 \leq \psi_{j} \leq 1, \quad \psi_{j}=1 \text { on } \overline{\Omega_{j}} \backslash Q_{1}, \quad \psi_{j}=0 \text { on } Q_{0} .
$$

We define the $\mathcal{C}^{1}$ vector field on $\Omega$ :

$$
h(x)= \begin{cases}\psi_{j}(x) m_{j}(x) & \text { if } x \in \Omega_{j} \\ 0 & \text { if } x \in \Omega \backslash \cup_{j} \Omega_{j}\end{cases}
$$

Proceeding as in [7], we multiply (4.22) by $h \cdot \nabla u$ and integrate on each $(S, T) \times \Omega_{j}$

$$
\int_{S}^{T} \int_{\Omega_{j}} h(x) \cdot \nabla u\left(u^{\prime \prime}+\Delta^{2} u-f\right) \mathrm{d} x \mathrm{~d} t=0
$$

For the sake of concision, we will omit the $\mathrm{d} x \mathrm{~d} t$ in the following integrals. This gives, after integrations by parts

$$
\begin{aligned}
\int_{S}^{T} \int_{\Gamma_{j}} & {\left[\frac{1}{2} h \cdot \nu_{j}\left(\left|u^{\prime}\right|^{2}-|\Delta u|^{2}\right)+\Delta u \frac{\partial(h \cdot \nabla u)}{\partial \nu}-h \cdot \nabla u \frac{\partial \Delta u}{\partial \nu}\right] } \\
= & {\left[\int_{\Omega_{j}} u^{\prime} h \cdot \nabla u\right]_{S}^{T}+\int_{S}^{T} \int_{\Omega_{j}}\left(\frac{1}{2} \operatorname{div} h\left(u^{\prime 2}-|\Delta u|^{2}\right)+\Delta h_{k} \frac{\partial u}{\partial x_{k}} \Delta u+2 \nabla h_{k} \cdot \nabla\left(\frac{\partial u}{\partial x_{k}}\right) \Delta u-f h \cdot \nabla u\right) . }
\end{aligned}
$$

Thanks to the choice of $\psi_{j}$, only the boundary term on $\left(\Gamma_{j} \backslash \gamma_{j}\left(x_{j}\right)\right) \cap \Gamma$ is nonvanishing in the left hand side of (4.24). But on this part of the boundary, we claim that $\partial_{\nu}(h \cdot \nabla u)=h \cdot \nu \Delta u$ (see also [20,21]). For this, we first remark that $u=0=\partial_{\nu} u$ there. Hence, $\partial_{i} u=0$ for $1 \leq i \leq N$ on $\left(\Gamma_{j} \backslash \gamma_{j}\left(x_{j}\right)\right) \cap \Gamma$, and we have

$$
\partial_{\nu}(h \cdot \nabla u)=\sum_{j=1}^{N} \partial_{j}\left(\sum_{i}^{N} h_{i} \partial_{i} u\right) \nu_{j}=\sum_{i, j} \partial_{j}\left(h_{i} \partial_{i} u\right) \nu_{j}=\sum_{i, j} h_{i} \partial_{i j} u \nu_{j} .
$$

Setting $v=\partial_{j} u$, and recalling that $\nabla u=0$ on $\left(\Gamma_{j} \backslash \gamma_{j}\left(x_{j}\right)\right) \cap \Gamma$, we have $\nabla v=\partial_{\nu} v \nu$. Hence, $\partial_{i} v=\sum_{k=1}^{N} \partial_{k} v \nu_{k} \nu_{i}$ for all $1 \leq i \leq N$. Coming back to $\partial_{j} u$, we obtain

$$
\partial_{i j} u=\sum_{k} \partial_{k j} u \nu_{k} \nu_{i}
$$

and in particular

$$
\partial_{j j} u=\sum_{k} \partial_{k j} u \nu_{k} \nu_{j} .
$$

Using (4.26) in (4.25), we deduce that

$$
\partial_{\nu}(h \cdot \nabla u)=\sum_{i, j, k} h_{i} \partial_{k j} u \nu_{k} \nu_{i} \nu_{j}=\sum_{i} h_{i} \nu_{i} \sum_{j}\left[\sum_{k}\left(\partial_{k j} u \nu_{k}\right) \nu_{j}\right] .
$$

Using (4.27) in this last identity, we obtain

$$
\partial_{\nu}(h \cdot \nabla u)=\sum_{i} h_{i} \nu_{i} \sum_{j} \partial_{j j} u=h \cdot \nu \Delta u,
$$


which proves our claim. Since in addition, $u^{\prime}=0$ on $\left(\Gamma_{j} \backslash \gamma_{j}\left(x_{j}\right)\right) \cap \Gamma$, we deduce that the left hand side of (4.24) reduces to

$$
\frac{1}{2} \int_{S}^{T} \int_{\left(\Gamma_{j} \backslash \gamma_{j}\left(x_{j}\right)\right) \cap \Gamma} h \cdot \nu|\Delta u|^{2} \leq 0 .
$$

Therefore, since $\psi_{j}=0$ on $Q_{0}$ and thanks to the above inequality used in (4.24), we deduce that

$$
\left[\int_{\Omega_{j}} u^{\prime} h \cdot \nabla u\right]_{S}^{T}+\int_{S}^{T} \int_{\Omega_{j} \backslash Q_{0}}\left(\frac{1}{2} \operatorname{div} h\left(u^{\prime 2}-|\Delta u|^{2}\right)+\Delta h_{k} \frac{\partial u}{\partial x_{k}} \Delta u+2 \nabla h_{k} \cdot \nabla\left(\frac{\partial u}{\partial x_{k}}\right) \Delta u-f h \cdot \nabla u\right) \leq 0 .
$$

Using $\psi_{j}=1$ on $\overline{\Omega_{j}}-Q_{1}$ and summing the resulting inequalities on $j$, we obtain

$$
\begin{aligned}
& {\left[\int_{\Omega} u^{\prime} h \cdot \nabla u\right]_{S}^{T} }+\int_{S}^{T} \int_{\Omega \backslash Q_{1}} \frac{1}{2}\left(N u^{\prime 2}+(2-N)|\nabla u|^{2}\right)-\int_{S}^{T} \int_{\Omega} f h \cdot \nabla u \\
& \leq-\sum_{j} \int_{S}^{T}\left[\int_{\Omega_{j} \cap Q_{1}} \frac{1}{2} \operatorname{div} h\left(u^{\prime 2}-|\Delta u|^{2}\right)+\Delta h_{k} \frac{\partial u}{\partial x_{k}} \Delta u+2 \nabla h_{k} \cdot \nabla\left(\frac{\partial u}{\partial x_{k}}\right) \Delta u\right] \\
& \leq C \int_{S}^{T} \int_{\Omega \cap Q_{1}}\left(u^{\prime 2}+|\Delta u|^{2}+|\nabla u|^{2}+\frac{\partial^{2} u}{\partial x_{i} \partial x_{k}} \frac{\partial^{2} u}{\partial x_{i} \partial x_{k}}\right),
\end{aligned}
$$

where $C$ is a positive constant which depends only on $\psi_{j}$ and $m_{j}$. We now use the second multiplier $u(N-2) / 2$ and evaluate the term

$$
\frac{N-2}{2} \int_{S}^{T} \int_{\Omega} u\left(u^{\prime \prime}+\Delta u^{2}-f\right)=0 .
$$

Hence, one has

$$
\frac{N-2}{2}\left[\int_{\Omega} u u^{\prime}\right]_{S}^{T}+\frac{N-2}{2} \int_{S}^{T} \int_{\Omega}|\Delta u|^{2}-u^{\prime 2}-u f=0
$$

We set $M(u)=h \cdot \nabla u+\frac{N-2}{2} u$. Adding (4.31) to (4.30), we obtain

$$
2 \int_{S}^{T} e \mathrm{~d} t \leq C \int_{S}^{T} \int_{\Omega \cap Q_{1}}\left[u^{\prime 2}+|\Delta u|^{2}+|\nabla u|^{2}+\frac{\partial^{2} u}{\partial x_{i} \partial x_{k}} \frac{\partial^{2} u}{\partial x_{i} \partial x_{k}}\right]-\left[\int_{\Omega} M(u) u^{\prime}\right]_{S}^{T}+\int_{S}^{T} \int_{\Omega} M(u) f .
$$

We estimate the terms on the right hand side of (4.32) as follows. First, we have

$$
\left|\left[\int_{\Omega} M(u) u^{\prime}\right]_{S}^{T}\right| \leq C(e(S)+e(T)) .
$$

Second, we estimate the last term of (4.32) as follows

$$
\left|\int_{S}^{T} \int_{\Omega} M(u) f\right| \leq \frac{C}{\mu} \int_{S}^{T} \int_{\Omega}|f|^{2}+\mu \int_{S}^{T} e \mathrm{~d} t \quad \forall \mu>0
$$

Using (4.33) and (4.34) in (4.32), we obtain for all $\mu>0$ :

$$
\begin{array}{r}
(2-\mu) \int_{S}^{T} e \mathrm{~d} t \leq C \int_{S}^{T} \int_{\Omega \cap Q_{1}}\left[u^{\prime 2}+|\nabla u|^{2}+|\Delta u|^{2}+\frac{\partial^{2} u}{\partial x_{i} \partial x_{k}} \frac{\partial^{2} u}{\partial x_{i} \partial x_{k}}\right] \\
+C(e(S)+e(T))+\frac{C}{\mu} \int_{S}^{T} \int_{\Omega}|f|^{2} .
\end{array}
$$


We now have to estimate the first term on the right hand side of (4.35), that we will denote by $X$ :

$$
X=\int_{S}^{T} \int_{\Omega \cap Q_{1}}\left[u^{\prime 2}+|\nabla u|^{2}+|\Delta u|^{2}+\frac{\partial^{2} u}{\partial x_{i} \partial x_{k}} \frac{\partial^{2} u}{\partial x_{i} \partial x_{k}}\right] .
$$

This is where the piecewise multiplier method takes its place.

\section{Step 1. Estimate of the terms corresponding to second derivatives in space in $X$.}

Since $\overline{\mathbb{R}^{N} \backslash Q_{2}} \cap \overline{Q_{1}}=\emptyset$, there exists a function $\xi \in \mathcal{C}_{0}^{\infty}\left(\mathbb{R}^{N}\right)$ such that

$$
0 \leq \xi \leq 1, \quad \xi=1 \text { on } Q_{1}, \quad \xi=0 \text { on } \mathbb{R}^{N} \backslash Q_{2} .
$$

We need the following result, that is proved in [7], Proposition 4.1.

Lemma 4.9. Let $\xi$ be defined as above. Then for all $v \in H_{0}^{2}(\Omega)$, we have

$$
\int_{\Omega} \nabla \xi \cdot \nabla v \Delta v=-\int_{\Omega} \frac{\partial^{2} \xi}{\partial x_{i} \partial x_{k}} \frac{\partial v}{\partial x_{i}} \frac{\partial v}{\partial x_{k}}+\frac{1}{2} \int_{\Omega} \Delta \xi|\nabla v|^{2}
$$

and

$$
\int_{\Omega} \xi \frac{\partial^{2} v}{\partial x_{i} \partial x_{k}} \frac{\partial^{2} v}{\partial x_{i} \partial x_{k}}=\int_{\Omega}\left[-\frac{\partial^{2} \xi}{\partial x_{i} \partial x_{k}} \frac{\partial v}{\partial x_{i}} \frac{\partial v}{\partial x_{k}}+\Delta \xi|\nabla v|^{2}+\xi|\Delta v|^{2}\right] .
$$

From (4.37), we deduce that

$$
\int_{\Omega \cap Q_{1}} \frac{\partial^{2} u}{\partial x_{i} \partial x_{k}} \frac{\partial^{2} u}{\partial x_{i} \partial x_{k}} \leq \int_{\Omega} \xi|\Delta u|^{2}+C \int_{\Omega \cap Q_{2}}|\nabla u|^{2} .
$$

We use now the multiplier $\xi u$ in the first equation of (4.22), and consider the expression:

$$
\int_{S}^{T} \int_{\Omega}\left(u^{\prime \prime}+\Delta^{2} u-f\right) \xi u \mathrm{~d} x \mathrm{~d} t=0
$$

After integrations by parts, this gives

$$
\int_{S}^{T} \int_{\Omega} \xi|\Delta u|^{2}=\int_{S}^{T} \int_{\Omega} \xi\left|u^{\prime}\right|^{2}-2 \nabla \xi \cdot \nabla u \Delta u-u \Delta u \Delta \xi-\left[\int_{\Omega} \xi u u^{\prime}\right]_{S}^{T}+\int_{S}^{T} \int_{\Omega} \xi u f .
$$

Using (4.36) in this last identity, we obtain

$$
\int_{S}^{T} \int_{\Omega} \xi|\Delta u|^{2}=\int_{S}^{T} \int_{\Omega}\left[\xi\left|u^{\prime}\right|^{2}+2 \frac{\partial^{2} \xi}{\partial x_{i} \partial x_{k}} \frac{\partial u}{\partial x_{i}} \frac{\partial u}{\partial x_{k}}+\Delta \xi|\nabla u|^{2}+u \Delta u \Delta \xi\right]-\left[\int_{\Omega} \xi u u^{\prime}\right]_{S}^{T}+\int_{S}^{T} \int_{\Omega} \xi u f .
$$

On the other hand, one has $\int_{\Omega} u \Delta u \Delta \xi=\int_{\Omega}\left(\frac{u^{2}}{2} \Delta^{2} \xi-|\nabla u|^{2} \Delta \xi\right)$. Using this identity in (4.39), we obtain

$$
\int_{S}^{T} \int_{\Omega} \xi|\Delta u|^{2}=\int_{S}^{T} \int_{\Omega}\left[\xi\left|u^{\prime}\right|^{2}+2 \frac{\partial^{2} \xi}{\partial x_{i} \partial x_{k}} \frac{\partial u}{\partial x_{i}} \frac{\partial u}{\partial x_{k}}-\frac{u^{2}}{2} \Delta^{2} \xi\right]-\left[\int_{\Omega} \xi u u^{\prime}\right]_{S}^{T}+\int_{S}^{T} \int_{\Omega} \xi u f .
$$

We estimate the second term on the right hand side of the above inequality as previously (see (4.33)). Moreover, since $\xi=1$ on $Q_{1}$, whereas $\xi=0$ on $\mathbb{R}^{N} \backslash Q_{2}$, we deduce that

$$
\int_{S}^{T} \int_{\Omega} \xi|\Delta u|^{2} \leq C(e(S)+e(T))+C \int_{S}^{T} \int_{\Omega \cap Q_{2}}\left|u^{\prime}\right|^{2}+|\nabla u|^{2}+u^{2}+|f|^{2} .
$$


Using (4.40) in (4.38), we obtain that

$$
\int_{\Omega \cap Q_{1}} \frac{\partial^{2} u}{\partial x_{i} \partial x_{k}} \frac{\partial^{2} u}{\partial x_{i} \partial x_{k}} \leq C(e(S)+e(T))+C \int_{S}^{T} \int_{\Omega \cap Q_{2}}\left|u^{\prime}\right|^{2}+|\nabla u|^{2}+u^{2}+|f|^{2},
$$

and

$$
\int_{S}^{T} \int_{\Omega \cap Q_{1}}|\Delta u|^{2} \leq C(e(S)+e(T))+C \int_{S}^{T} \int_{\Omega \cap Q_{2}}\left|u^{\prime}\right|^{2}+|\nabla u|^{2}+u^{2}+|f|^{2} .
$$

Using both (4.41) and (4.42) in (4.35), we obtain for all $\mu>0$

$$
(2-\mu) \int_{S}^{T} e \mathrm{~d} t \leq C \int_{S}^{T} \int_{\Omega \cap Q_{2}}\left[u^{\prime 2}+|\nabla u|^{2}+|u|^{2}\right]+C(e(S)+e(T))+C\left(1+\frac{1}{\mu}\right) \int_{S}^{T} \int_{\Omega}|f|^{2} .
$$

\section{Step 2. Estimate of the terms corresponding to first derivatives in space in $X$.}

Since $\overline{\mathbb{R}^{N} \backslash Q_{3}} \cap \overline{Q_{2}}=\emptyset$, there exists a function $\beta \in \mathcal{C}_{0}^{\infty}\left(\mathbb{R}^{N}\right)$ such that

$$
0 \leq \beta \leq 1, \quad \beta=1 \text { on } Q_{2}, \quad \beta=0 \text { on } \mathbb{R}^{N} \backslash Q_{3} .
$$

We fix $t$ and consider the solution $\theta$ of the following elliptic problem:

$$
\begin{cases}\Delta^{2} \theta=\beta \Delta u & \text { in } \Omega, \\ \theta=0=\frac{\partial \theta}{\partial \nu} & \text { on } \Gamma .\end{cases}
$$

Then, we have

$$
\int_{\Omega}|\Delta \theta|^{2}=\int_{\Omega} \theta \Delta^{2} \theta=\int_{\Omega} u \Delta(\beta \theta)=\int_{\Omega \cap Q_{3}} u(\beta \Delta \theta+2 \nabla \beta \cdot \nabla \theta+\theta \Delta \beta) \leq C \int_{\Omega^{\prime} Q_{3}}|u|^{2},
$$

and similarly,

$$
\int_{\Omega}\left|\Delta \theta^{\prime}\right|^{2} \leq C \int_{\Omega \cap Q_{3}}\left|u^{\prime}\right|^{2}
$$

We now consider the multiplier $\theta$ for (4.22) and evaluate the expression

$$
\int_{S}^{T} \int_{\Omega} \theta\left(u^{\prime \prime}+\Delta u^{2}-f\right)=0
$$

This yields, after integrations by parts,

$$
\int_{S}^{T} \int_{\Omega} \beta u \Delta u-\int_{S}^{T} \int_{\Omega} \theta^{\prime} u^{\prime}+\left[\int_{\Omega} \theta u^{\prime}\right]_{S}^{T}-\int_{S}^{T} \int_{\Omega} \theta f=0
$$

Integrating by parts the first term of this expression, we obtain

$$
\int_{S}^{T} \int_{\Omega} \beta|\nabla u|^{2}=-\int_{S}^{T} \int_{\Omega} \theta^{\prime} u^{\prime}+\int_{S}^{T} \int_{\Omega} \frac{\Delta \beta}{2}|u|^{2}+\left[\int_{\Omega} \theta u^{\prime}\right]_{S}^{T}+\int_{S}^{T} \int_{\Omega} \theta f .
$$

Since $\beta=1$ on $\Omega \cap Q_{2}$, whereas $\beta=0$ on $\Omega \backslash Q_{3}$, and thanks to (4.43), we obtain for all $\mu>0$ :

$$
\int_{S}^{T} \int_{\Omega \cap Q_{2}} \beta|\nabla u|^{2} \leq \frac{\mu}{2} \int_{S}^{T} \int_{\Omega}\left|u^{\prime}\right|^{2}+\frac{1}{2 \mu} \int_{S}^{T} \int_{\Omega}\left|\theta^{\prime}\right|^{2}+C \int_{S}^{T} \int_{\Omega \cap Q_{3}}|u|^{2}+C \int_{S}^{T} \int_{\Omega}|f|^{2}+C(e(S)+e(T)) .
$$


Using, (4.44) in this last inequality, we deduce that, for all $\mu>0$

$$
\int_{S}^{T} \int_{\Omega \cap Q_{2}}|\nabla u|^{2} \leq \mu \int_{S}^{T} e+\frac{C}{\mu} \int_{S}^{T} \int_{\Omega \cap Q_{3}}\left|u^{\prime}\right|^{2}+C \int_{S}^{T} \int_{\Omega \cap Q_{3}}|u|^{2}+C \int_{S}^{T} \int_{\Omega}|f|^{2}+C(e(S)+e(T)) .
$$

Finally, putting this into (4.43), we obtain for all $\mu>0$

$$
\begin{aligned}
(2-C \mu) \int_{S}^{T} e \mathrm{~d} t \leq C \int_{S}^{T} \int_{\Omega \cap Q_{3}}|u|^{2}+C\left(1+\frac{1}{\mu}\right) \int_{S}^{T} \int_{\Omega \cap Q_{3}} u^{\prime 2} & \\
& +C\left(1+\frac{1}{\mu}\right) \int_{S}^{T} \int_{\Omega}|f|^{2}+C(e(S)+e(T)) .
\end{aligned}
$$

\section{Step 3. Estimate of the zero order terms in $X$.}

Since $\overline{\mathbb{R}^{N} \backslash \omega} \cap \overline{Q_{3}}=\emptyset$, there exists a function $\psi \in \mathcal{C}_{0}^{\infty}\left(\mathbb{R}^{N}\right)$ such that

$$
0 \leq \psi \leq 1, \quad \psi=1 \text { on } Q_{3}, \quad \psi=0 \text { on } \mathbb{R}^{N} \backslash \omega .
$$

We fix $t$ and consider the solution $z$ of the following elliptic problem

$$
\begin{cases}\Delta^{2} z=\psi u & \text { in } \Omega, \\ z=0=\frac{\partial z}{\partial \nu} & \text { on } \Gamma .\end{cases}
$$

Then, we have

$$
\int_{\Omega}|\Delta z|^{2} \leq C \int_{\omega} \psi|u|^{2}, \quad \text { and } \quad \int_{\Omega}\left|\Delta z^{\prime}\right|^{2} \leq C \int_{\omega}\left|u^{\prime}\right|^{2} .
$$

We now consider the multiplier $z$ for (4.22) and evaluate the expression

$$
\int_{S}^{T} \int_{\Omega} z\left(u^{\prime \prime}+\Delta u^{2}-f\right)=0
$$

This yields

$$
\int_{S}^{T} \int_{\Omega} \psi|u|^{2}-\int_{S}^{T} \int_{\Omega} z^{\prime} u^{\prime}+\left[\int_{\Omega} z u^{\prime}\right]_{S}^{T}-\int_{S}^{T} \int_{\Omega} z f=0 .
$$

Integrating by parts the first term of this expression, we obtain

$$
\int_{S}^{T} \int_{\Omega} \psi|u|^{2}=\int_{S}^{T} \int_{\Omega} z^{\prime} u^{\prime}-\left[\int_{\Omega} z u^{\prime}\right]_{S}^{T}+\int_{S}^{T} \int_{\Omega} z f
$$

Hence, using (4.46) to estimate the third and fourth terms on the right hand side of the above equality, we obtain for all $\eta>0, \mu>0$ :

$$
\int_{S}^{T} \int_{\Omega} \psi|u|^{2} \leq \frac{\mu}{2} \int_{S}^{T} \int_{\Omega}\left|u^{\prime}\right|^{2}+\eta \int_{S}^{T} \int_{\Omega}|z|^{2}+\frac{c}{\mu} \int_{S}^{T} \int_{\Omega}\left|z^{\prime}\right|^{2}+\frac{c}{\eta} \int_{S}^{T} \int_{\Omega}|f|^{2}+C(e(S)+e(T)) .
$$

Using now the definition of the energy, together with (4.46), we deduce that

$$
(1-c \eta) \int_{S}^{T} \int_{\Omega} \psi|u|^{2} \leq \mu \int_{S}^{T} e+\frac{c}{\mu} \int_{S}^{T} \int_{\omega}\left|u^{\prime}\right|^{2}+\frac{c}{\eta} \int_{S}^{T} \int_{\Omega}|f|^{2}+C(e(S)+e(T)) .
$$


As a consequence, since $\psi=1$ on $Q_{3}$ and choosing $\eta$ sufficiently small, we have for all $\mu>0$ :

$$
\int_{S}^{T} \int_{\Omega \cap Q_{3}}|u|^{2} \leq c \mu \int_{S}^{T} e+\frac{c}{\mu} \int_{S}^{T} \int_{\omega}\left|u^{\prime}\right|^{2}+c \int_{S}^{T} \int_{\Omega}|f|^{2}+C(e(S)+e(T)) .
$$

Using this last estimate in (4.45), we obtain for all $\mu>0$

$$
(2-c \mu) \int_{S}^{T} e \mathrm{~d} t \leq C\left(1+\frac{1}{\mu}\right) \int_{S}^{T} \int_{\omega}\left|u^{\prime}\right|^{2}+C\left(1+\frac{1}{\mu}\right) \int_{S}^{T} \int_{\Omega}|f|^{2}+C(e(S)+e(T)) .
$$

Finally, choosing now $\mu$ sufficiently small, we have

$$
\int_{S}^{T} e \mathrm{~d} t \leq C(e(S)+e(T))+C \int_{S}^{T} \int_{\Omega}|f|^{2}+C \int_{S}^{T} \int_{\omega}\left|u^{\prime}\right|^{2}
$$

and the lemma is proved.

Acknowledgements. The second author wishes to thank O. Glass, J. Le Rousseau, and L. Robbiano for very fruitful discussions and encouragements. Both authors were partially supported by "l'Agence Nationale de la Recherche" under grant ANR-07-JCJC-0139-01 and the GDRE CONEDP (CNRS/INDAM/UP). We are grateful to the referees for their valuable comments and suggestions.

\section{REFERENCES}

[1] F. Alabau, P. Cannarsa and V. Komornik, Indirect internal damping of coupled systems. J. Evol. Equ. 2 (2002) 127-150.

[2] F. Alabau-Boussouira, Stabilisation frontière indirecte de systèmes faiblement couplés. C. R. Acad. Sci. Paris Sér. I Math. 328 (1999) 1015-1020.

[3] F. Alabau-Boussouira, Indirect boundary stabilization of weakly coupled systems. SIAM J. Control Optim. 41 (2002) 511-541.

[4] F. Alabau-Boussouira, A two-level energy method for indirect boundary observability and controllability of weakly coupled hyperbolic systems. SIAM J. Control Optim. 42 (2003) 871-906.

[5] F. Alabau-Boussouira, Une formule générale pour le taux de décroissance des systèmes dissipatifs non linéaires. $C$. $R$. Acad. Sci. Paris Sér. I Math. 338 (2004) 35-40.

[6] F. Alabau-Boussouira, Convexity and weighted integral inequalities for energy decay rates of nonlinear dissipative hyperbolic systems. Appl. Math. Optim. 51 (2005) 61-105.

[7] F. Alabau-Boussouira, Piecewise multiplier method and nonlinear integral inequalities for Petrowsky equation with nonlinear dissipation. J. Evol. Equ. 6 (2006) 95-112.

[8] F. Alabau-Boussouira, Asymptotic behavior for Timoshenko beams subject to a single nonlinear feedback control. NoDEA 14 (2007) 643-669.

[9] F. Ammar-Khodja, A. Benabdallah, J.E. Muñoz Rivera and R. Racke, Energy decay for Timoshenko systems of memory type. J. Differ. Equ. 194 (2003) 82-115.

[10] F. Ammar-Khodja, A. Benabdallah and C. Dupaix, Null controllability of some reaction-diffusion systems with one control force. J. Math. Anal. Appl. 320 (2006) 928-943.

[11] C. Bardos, G. Lebeau and J. Rauch, Sharp sufficient conditions for the observation, control, and stabilization of waves from the boundary. SIAM J. Control Optim. 30 (1992) 1024-1065.

[12] A. Beyrath, Indirect linear locally distributed damping of coupled systems. Bol. Soc. Parana. Math. 22 (2004) 17-34.

[13] N. Burq, Décroissance de l'énergie locale de l'équation des ondes pour le problème extérieur et absence de résonance au voisinage du réel. Acta Math. 180 (1998) 1-29.

[14] N. Burq and G. Lebeau, Mesures de défaut de compacité, application au système de Lamé. Ann. Sci. Éc. Norm. Supér. 34 (2001) 817-870.

[15] F. Conrad and B. Rao, Decay of solutions of the wave equation in a star-shaped domain with nonlinear boundary feedback. Asymptotic Anal. 7 (1993) 159-177.

[16] L. de Teresa, Insensitizing controls for a semilinear heat equation. Commun. Part. Differ. Equ. 25 (2000) 39-72.

[17] M. González-Burgos and R. Pérez-García, Controllability results for some nonlinear coupled parabolic systems by one control force. Asymptotic Anal. 46 (2006) 123-162.

[18] A. Haraux, Semi-groupes linéaires et équations d'évolution linéaires périodiques. Publication du Laboratoire d’Analyse Numérique, Université Pierre et Marie Curie 78011 (1978) 123-162. 
[19] O. Kavian and L. de Teresa, Unique continuation principle for systems of parabolic equations. ESAIM: COCV 16 (2010) $247-274$.

[20] V. Komornik, Exact controllability and stabilization: The multiplier method. Research in Applied Mathematics 36, Masson, Paris (1994).

[21] J.E. Lagnese, Boundary stabilization of thin plates, SIAM Studies in Applied Mathematics 10. SIAM (1989).

[22] M. Léautaud, Spectral inequalities for non-selfadjoint elliptic operators and application to the null-controllability of parabolic systems. J. Funct. Anal. 258 (2010) 2739-2778.

[23] G. Lebeau, Équation des ondes amorties, in Algebraic and geometric methods in mathematical physics. Math. Phys. Stud., Kluwer Acad. Publ., Dordrecht (1996) 73-109.

[24] G. Lebeau and L. Robbiano, Stabilisation de l'équation des ondes par le bord. Duke Math. J. 86 (1997) $465-491$.

[25] G. Lebeau and E. Zuazua, Decay rates for the three-dimensional linear system of thermoelasticity. Arch. Rational Mech. Anal. 148 (1999) 179-231.

[26] J.-L. Lions, Contrôlabilité exacte, perturbations et stabilisation de systèmes distribués, Recherches en Mathématiques Appliquées, Tome 1, 8. Masson, Paris (1988).

[27] K. Liu, Locally distributed control and damping for the conservative systems. SIAM J. Control Optim. 35 (1997) 1574-1590.

[28] P. Martinez, A new method to obtain decay rate estimates for dissipative systems with localized damping. Rev. Math. Comput. 12 (1999) 251-283.

[29] L. Miller, Escape function conditions for the observation, control, and stabilization of the wave equation. SIAM J. Control Optim. 41 (2002) 1554-1566.

[30] W. Youssef, Contrôle et stabilisation de systèmes élastiques couplés. Ph. D. thesis, University of Metz, France (2009).

[31] E. Zuazua, Exponential decay for the semilinear wave equation with locally distributed damping. Commun. Part. Differ. Equ. 15 (1990) 205-235. 